\title{
EXISTENCE RESULTS FOR A CLASS OF ABSTRACT IMPULSIVE DIFFERENTIAL EQUATIONS
}

\author{
EDUARDO HERNÁNDEZ ${ }^{\bowtie}$ and DONAL O'REGAN
}

(Received 18 February 2012; accepted 8 December 2012; first published online 18 March 2013)

\begin{abstract}
We study the existence of solutions for a class of abstract impulsive differential equations. Our technical framework allows us to study partial differential equations with impulsive conditions involving partial derivatives and nonlinear expressions of the solution. Some applications to impulsive partial differential equations are presented.
\end{abstract}

2010 Mathematics subject classification: primary 34K45, 34K30, 35R12; secondary 47D06.

Keywords and phrases: impulsive differential equations, nonlinear impulse, analytic semigroup, nonlinear equations.

\section{1. introduction}

In this paper we study the existence of solutions for abstract impulsive differential equations of the form

$$
\begin{aligned}
u^{\prime}(t) & =A u(t)+f(t, u(t)), \quad t \in(0, a], t \neq t_{i}, i=1, \ldots, N, \\
u(0) & =x_{0} \in X, \\
\triangle u\left(t_{i}\right) & =I_{i}\left(u\left(t_{i}\right)\right), \quad i=1, \ldots, N,
\end{aligned}
$$

where $A: D(A) \subset X \rightarrow X$ is the infinitesimal generator of an analytic semigroup of bounded linear operators $(T(t))_{t \geq 0}$ defined on a Banach space $(X,\|\cdot\|), x_{0} \in X, 0<$ $t_{1}<\cdots<t_{N}<a$ are fixed numbers, $I_{i} \in C\left(X_{\alpha}, X\right)$ for all $i=1, \ldots, N, X_{\alpha}$ denotes the domain of the $\alpha$-fractional power of $-A$ endowed with the graph norm $\left\|(-A)^{\alpha} x\right\|$, $f:[0, a] \times X_{\alpha} \rightarrow X$ is a suitable function and $\Delta u\left(t_{i}\right)$ represents the jump of $u(\cdot)$ at $t_{i}$ which is defined by $\Delta u\left(t_{i}\right)=u\left(t_{i}^{+}\right)-u\left(t_{i}^{-}\right)$.

The literature on abstract impulsive differential equations similar to (1.1)-(1.3) is very extensive and considers different topics on the existence and qualitative properties of solutions. Concerning general motivations, relevant developments and the current status of the theory we refer the reader to $[1-19,21,22]$ and the references therein.

(C) 2013 Australian Mathematical Publishing Association Inc. 0004-9727/2013 \$16.00 
In comparison to the literature on abstract impulsive differential equations, the main contribution of our paper is the type of function $I_{i}$ considered in (1.3). In this work we assume that the maps $I_{i}$ are defined from $X_{\alpha}$ into $X$, which permit the study of partial differential equations with impulsive conditions involving partial derivatives and nonlinear expressions of the solution (see the examples in the final section). It is important to note that in $[2,5,6,9,15,21]$ some results are established on the existence of $X_{\alpha}$-valued mild solutions for different classes of impulsive differential equations. However, in these papers the maps $I_{i}$ are defined from $X_{\alpha}$ into $X_{\alpha}$, and do not permit the applications mentioned.

This paper has three sections. In Section 2 we study the existence of mild and classical solutions for (1.1)-(1.3). To prove our results on the existence of a mild solution (see Definition 2.2), we need to look at the singularity of the function $t \mapsto\left\|T\left(t-t_{i}\right) I_{i}\left(u\left(t_{i}\right)\right)\right\|_{\alpha}$ at $t=t_{t_{i}}$. To this end, we introduce a class of Banach spaces with weight (denoted $\mathcal{P} C_{\alpha}\left(X_{\alpha}\right)$ ) formed by piecewise continuous functions for which $\sup _{t \in\left(t_{i}, t_{i+1}\right]}\left(t-t_{i}\right)^{\alpha}\|u(t)\|_{\alpha}$ is finite. The existence and uniqueness of a mild solution via the contraction mapping principle is established in Theorem 2.6. In the main result of this paper (Theorem 2.13) we prove the existence of a mild solution via Schauder's fixed point criteria without assuming that the functions $I_{i}$ are compact. To prove this theorem we use the approximation scheme of Fan and Li [8], but in the context of the space $\mathcal{P} C_{\alpha}\left(X_{\alpha}\right)$. First, we study the solvability of a family of integral equations (see equation (2.6)) which are a 'good approximation' of the integral equation used to define the concept of mild solution of (1.1)-(1.3). In Theorem 2.9, we prove the existence of a solution $u_{n}, n \in \mathbb{N}$, for (2.6) and in Lemmas 2.11 and 2.12 we establish some compactness properties of the set $\left\{u_{n}: n \in \mathbb{N}\right\}$. Finally, by proving the existence of a convergent subsequence of $\left(u_{n}\right)_{n \in \mathbb{N}}$, in Theorem 2.13 we show the existence of a mild solution for (1.1)-(1.3). We finish Section 2 by studying the existence of a classical solution for (1.1)-(1.3). In the final section, some applications to partial differential equations with impulsive terms involving partial derivatives and nonlinear expressions of the solution are considered.

Next, we introduce some definitions, notation and results used in this work. Let $\left(Z,\|\cdot\|_{Z}\right)$ and $\left(W,\|\cdot\|_{W}\right)$ be Banach spaces. We denote by $\mathcal{L}(Z, W)$ the space of bounded linear operators from $Z$ into $W$ endowed with the norm of operators denoted by $\|\cdot\|_{\mathcal{L}(Z, W)}$ and we write $\mathcal{L}(Z)$ and $\|\cdot\|_{\mathcal{L}(Z)}$ when $Z=W$. In addition, $B_{l}(z, Z)$ denotes the closed ball with centre at $z \in Z$ and radius $l$ in $Z$ and for $J \subset \mathbb{R}$ we use the notation $C(J ; Z)$ for the space formed by all the bounded continuous functions from $J$ into $Z$ endowed with the uniform norm denoted by $\|\cdot\|_{C(J ; X)}$. In this work, for a function $u: J \rightarrow X$ and $I \subset J$, the symbol $u_{\left.\right|_{I}}$ represents the restriction of $u(\cdot)$ to the set $I$.

In this paper, $A: D(A) \subset X \rightarrow X$ is the generator of an analytic semigroup $(T(t))_{t \geq 0}$ on $(X,\|\cdot\|)$ and we assume that $0 \in \rho(A)$. From [20] we note that $X_{\gamma}$ is a Banach space, $X_{\gamma} \hookrightarrow X_{\beta}$ for $0<\beta<\gamma<\infty,(-A)^{\beta} T(\cdot) \in C((0, \infty), \mathcal{L}(X))$ and there is $C_{\beta}>0$ such that $\left\|(-A)^{\beta} T(t)\right\|_{\mathcal{L}(X)} \leq C_{\beta} t^{-\beta}\|x\|$ for all $t \in(0, a]$.

In the remainder of this paper, $\alpha \in(0,1), t_{0}=0, t_{N+1}=a$ and $\delta_{i}=\left(t_{i+1}-t_{i}\right)$ for all $i=0, \ldots, N$. To treat the impulsive conditions, we consider the space $\mathcal{P} C(X)$ formed 
by all the functions $u:[0, a] \rightarrow X$ such that $u(\cdot)$ is continuous at $t \neq t_{i}, u\left(t_{i}^{-}\right)=u\left(t_{i}\right)$ and $u\left(t_{i}^{+}\right)$exists for all $i=1, \ldots, N$, endowed with the uniform norm denoted by $\|\cdot\|_{\mathcal{P C}(X)}$. The notation $\mathcal{P} C_{\alpha}\left(X_{\alpha}\right)$ is used for the space formed by all the functions $u:(0, a] \rightarrow X_{\alpha}$ such that $u_{\left.\mid t_{i,}, t_{i+1}\right]} \in C\left(\left(t_{i}, t_{i+1}\right] ; X_{\alpha}\right)$ and $\|u\|_{\alpha, i}=\sup _{t \in\left(t_{i}, t_{i+1}\right]}\left(t-t_{i}\right)^{\alpha}\left\|(-A)^{\alpha} u(t)\right\|<\infty$ for all $i=0, \ldots, N$, endowed with the norm $\|u\|_{\alpha}=\max _{i=0,1, \ldots, N}\|u\|_{\alpha, i}$. It is easy to see that $\mathcal{P C}\left(X_{\alpha}\right)$ is a Banach space.

For a function $u \in \mathcal{P C}(X)$ and $i \in\{0,1, \ldots, N\}$, we use the notation $\tilde{u}_{i}$ for the function $\tilde{u}_{i} \in C\left(\left[t_{i}, t_{i+1}\right] ; X\right)$ given by

$$
\widetilde{u}_{i}(t)= \begin{cases}u(t) & \text { for } t \in\left(t_{i}, t_{i+1}\right] \\ u\left(t_{i}^{+}\right) & \text {for } t=t_{i}\end{cases}
$$

If $B \subseteq \mathcal{P C}(X)$, we employ the notation $\widetilde{B}_{i}$ for the set $\widetilde{B}_{i}=\left\{\tilde{u}_{i}: u \in B\right\}$. We state without proof the following Ascoli-Arzelà type criteria.

Lemma 1.1. A set $B \subseteq \mathcal{P C}(X)$ is relatively compact in $\mathcal{P C}(X)$ if and only if $\widetilde{B}_{i}$ is relatively compact in $C\left(\left[t_{i}, t_{i+1}\right], X\right)$ for all $i \in\{1, \ldots, N\}$.

Lemma 1.2. Assume that $K \subset \mathcal{P} C_{\alpha}\left(X_{\alpha}\right)$, the set $\left\{u_{\mid\left[b, t_{i+1}\right]}: u \in K\right\}$ is relatively compact in $C\left(\left[b, t_{i+1}\right], X_{\alpha}\right)$ for all $b \in\left(t_{i}, t_{i+1}\right]$ and each $i=0, \ldots, N$, and $\lim _{t \downarrow t_{i}} \sup _{u \in K}\left(t-t_{i}\right)^{\alpha}$ $\left\|(-A)^{\alpha} u(t)\right\|=0$ for all $i=1, \ldots, N$. Then $K$ is relatively compact in $\mathcal{P} C_{\alpha}\left(X_{\alpha}\right)$.

\section{Existence of solutions}

In this section we discuss the existence of solutions for the impulsive problem (1.1)(1.3). To begin, we introduce the following concepts of solution.

Definition 2.1. A function $u \in \mathcal{P C}(X)$ is called a classical solution of the problem

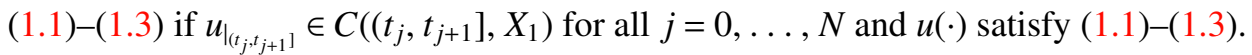

Definition 2.2. A function $u \in \mathcal{P C}(X)$ is called a mild solution of (1.1)-(1.3) if

$$
u(t)=T(t) x_{0}+\int_{0}^{t} T(t-s) f(s, u(s)) d s+\sum_{t_{i}<t} T\left(t-t_{i}\right) I_{i}\left(u\left(t_{i}\right)\right), \quad \forall t \in[0, a] .
$$

To prove our results, we introduce the following conditions. Let $q^{\prime}$ denote the conjugate of a number $q>1$ (that is, $1 / q+1 / q^{\prime}=1$ ) and take $q^{\prime}=\infty$ for $q=1$.

$H_{1} \quad$ The functions $I_{i}$ belong to $C\left(X_{\alpha} ; X\right)$ and there are nondecreasing functions $\mathcal{K}_{I_{i}}:[0, \infty) \rightarrow \mathbb{R}^{+}$such that $\left\|I_{i}(x)-I_{i}(y)\right\| \leq \mathcal{K}_{I_{i}}(l)\left\|(-A)^{\alpha}(x-y)\right\|$ for all $x, y \in$ $B_{l}\left(0, X_{\alpha}\right)$ and each $l>0$.

$H_{2} \quad$ For all $x \in X$, the function $f(\cdot, x)$ is strongly measurable on $[0, a]$ and $f(t, \cdot) \in C\left(X_{\alpha} ; X\right)$ for each $t \in[0, a]$. There are $q \in[1,1 / \alpha), m_{f} \in L^{q^{\prime}}\left([0, a] ; \mathbb{R}^{+}\right)$ and a nondecreasing function $W_{f} \in C\left([0, \infty) ; \mathbb{R}^{+}\right)$such that $\|f(t, x)\| \leq$ $m_{f}(t) W_{f}\left(\left\|(-A)^{\alpha} x\right\|\right)$ for all $(t, x) \in[0, a] \times X_{\alpha}$.

$H_{3}$ The functions $I_{i}$ belong to $C\left(X_{\alpha} ; X\right)$ and there are nondecreasing functions $c_{i}:[0, \infty) \rightarrow \mathbb{R}^{+}$and constants $d_{i}>0$ such that $\left\|I_{i}(x)\right\| \leq c_{i}(l)\left\|(-A)^{\alpha} x\right\|+d_{i}$ for all $x \in B_{l}\left(0, X_{\alpha}\right)$ and every $l>0$. 
$H_{4}$ The function $f$ belongs to $C\left([0, a] \times X_{\alpha} ; X\right)$ and there are $q \in[1,1 / \alpha)$ and a function $\mathcal{K}_{f} \in L^{q^{\prime}}\left([0, a] ; \mathbb{R}^{+}\right)$such that $\|f(t, x)-f(t, y)\| \leq \mathcal{K}_{f}(t) \|(-A)^{\alpha} x-$ $(-A)^{\alpha} y \|$ for all $x, y \in X_{\alpha}$ and every $t \in[0, a]$.

To shorten the proof of our existence results, we consider some lemmas.

Lemma 2.3. Assume that $\xi \in C\left((0, \infty) ; \mathbb{R}^{+}\right)$is nondecreasing, $\vartheta>0, \quad q>1, \quad \eta \in$ $L^{q^{\prime}}([0, a] ; \mathbb{R})$ and the function $\tau \rightarrow \xi^{q}\left(1 / \tau^{\alpha}\right)$ is integrable on $[0, a]$. Then

$$
\int_{c}^{d} \frac{\eta(\tau) \xi\left(\frac{\vartheta}{(\tau-c)^{\alpha}}\right)}{(d-\tau)^{\alpha}} d \tau \leq \frac{2^{\alpha+1 / q}}{(1-q \alpha)^{1 / q}} \frac{\|\eta\|_{L^{q^{\prime}}([c, d])}}{(d-c)^{\alpha}}\left(\int_{0}^{(d-c) / 2} \xi^{q}\left(\frac{2^{\alpha} \vartheta}{\tau^{\alpha}}\right) d \tau\right)^{1 / q}
$$

for all $0 \leq c<d \leq a$.

Proof. From the Hölder inequality, we see that

$$
\int_{c}^{d} \frac{\eta(\tau) \xi\left(\frac{\vartheta}{(\tau-c)^{\alpha}}\right)}{(d-\tau)^{\alpha}} d \tau \leq\|\eta\|_{L^{q^{\prime}([c, d])}}\left(\int_{0}^{d-c} \frac{\xi^{q}\left(\frac{\vartheta}{\tau^{\alpha}}\right)}{(d-c-\tau)^{q \alpha}} d \tau\right)^{1 / q}
$$

On the other hand, for $s \in[0, a]$,

$$
\begin{aligned}
\int_{0}^{s} \frac{\xi^{q}\left(\frac{\vartheta}{\tau^{\alpha}}\right)}{(s-\tau)^{q \alpha}} d \tau & \leq\left(\frac{s}{2}\right)^{-q \alpha} \int_{0}^{s / 2} \xi^{q}\left(\frac{\vartheta}{\tau^{\alpha}}\right) d \tau+\xi^{q}\left(\frac{\vartheta}{\left(\frac{s}{2}\right)^{\alpha}}\right) \int_{s / 2}^{s} \frac{d \tau}{(s-\tau)^{q \alpha}} \\
& \leq \frac{2^{q \alpha}}{s^{q \alpha}} \int_{0}^{s / 2} \xi^{q}\left(\frac{\vartheta}{\tau^{\alpha}}\right) d \tau+\xi^{q}\left(\frac{2^{\alpha} \vartheta}{s^{\alpha}}\right) \frac{\left(\frac{s}{2}\right)^{1-q \alpha}}{(1-q \alpha)} \\
& \leq \frac{2^{q \alpha}}{s^{q \alpha}} \int_{0}^{s / 2} \xi^{q}\left(\frac{2^{\alpha} \vartheta}{\tau^{\alpha}}\right) d \tau+\frac{\left(\frac{s}{2}\right)^{-q \alpha}}{(1-q \alpha)} \int_{0}^{s / 2} \xi^{q}\left(\frac{2^{\alpha} \vartheta}{s^{\alpha}}\right) d \tau
\end{aligned}
$$

from which we obtain that

$$
\int_{0}^{s} \frac{\xi^{q}\left(\frac{\vartheta}{\tau^{\alpha}}\right)}{(s-\tau)^{q \alpha}} d \tau \leq \frac{2^{1+q \alpha}}{s^{q \alpha}(1-q \alpha)} \int_{0}^{s / 2} \xi^{q}\left(\frac{2^{\alpha} \vartheta}{\tau^{\alpha}}\right) d \tau
$$

Now, the assertion follows by combining (2.1) and (2.2). The proof is complete.

Lemma 2.4. Assume that $\xi \in C\left((0, \infty) ; \mathbb{R}^{+}\right)$is nondecreasing, $\eta \in L^{q^{\prime}}([0, a] ; \mathbb{R})$ for some $q>1$, the function $\tau \rightarrow \xi^{q}\left(1 / \tau^{\alpha}\right)$ is integrable on $[0, a], u \in \mathcal{P} C_{\alpha}\left(X_{\alpha}\right)$ and $t \in$ $\left(t_{j}, t_{j+1}\right]$ for some $j \in\{1, \ldots, N\}$. Then

$$
\int_{0}^{t} \frac{\eta(s) \xi\left(\left\|(-A)^{\alpha} u(s)\right\|\right)}{(t-s)^{\alpha}} d s \leq \frac{\|\eta\|_{L^{q^{\prime}([0, t])}}}{\left(t-t_{j}\right)^{\alpha}}\left(N+\frac{2^{\alpha+1 / q}}{(1-q \alpha)^{1 / q}}\right)\left(\int_{0}^{a} \xi^{q}\left(\frac{2^{\alpha}\|u\|_{\alpha}}{s^{\alpha}}\right) d s\right)^{1 / q} .
$$


Proof. By noting that $\left\|(-A)^{\alpha} u(\tau)\right\| \leq\|u\|_{\alpha} /\left(\tau-t_{i}\right)^{\alpha}$ and $\left\|(-A)^{\alpha} u\left(t_{i}\right)\right\| \leq\|u\|_{\alpha} / \delta_{i-1}^{\alpha}$ for $i \in\{1, \ldots, N\}$ and $\tau \in\left(t_{i}, t_{i+1}\right]$, from Lemma 2.3 we have that

$$
\begin{aligned}
\int_{0}^{t} & \frac{\eta(s) \xi\left(\left\|(-A)^{\alpha} u(s)\right\|\right)}{(t-s)^{\alpha}} d s \\
\leq & \sum_{i=0}^{j-1} \int_{t_{i}}^{t_{i+1}} \frac{\eta(s)}{(t-s)^{\alpha}} \xi\left(\frac{\|u\|_{\alpha}}{\left(s-t_{i}\right)^{\alpha}}\right) d s+\int_{t_{j}}^{t} \frac{\eta(s)}{(t-s)^{\alpha}} \xi\left(\frac{\|u\|_{\alpha}}{\left(s-t_{j}\right)^{\alpha}}\right) d s \\
\leq & \frac{1}{\left(t-t_{j}\right)^{\alpha}} \sum_{i=0}^{j-1}\|\eta\|_{L^{q^{\prime}}\left(\left[t_{i}, t_{i+1}\right]\right)}\left(\int_{0}^{t_{i+1}-t_{i}} \xi^{q}\left(\frac{\|u\|_{\alpha}}{s^{\alpha}}\right) d s\right)^{1 / q} \\
& +\frac{2^{\alpha+1 / q}\|\eta\|_{L^{q^{\prime}}\left(\left[t_{j}, t\right]\right)}}{\left(t-t_{j}\right)^{\alpha}(1-q \alpha)^{1 / q}}\left(\int_{0}^{\left(t-t_{j}\right) / 2} \xi\left(\frac{2^{\alpha}\|u\|_{\alpha}}{s^{\alpha}}\right) d s\right)^{1 / q} \\
\leq & \frac{\|\eta\|_{L^{q^{\prime}}([0, t])}}{\left(t-t_{j}\right)^{\alpha}}\left(N+\frac{2^{\alpha+1 / q}}{(1-q \alpha)^{1 / q}}\right)\left(\int_{0}^{a} \xi\left(\frac{2^{\alpha}\|u\|_{\alpha}}{s^{\alpha}}\right) d s\right)^{1 / q}
\end{aligned}
$$

which completes the proof.

REMARK 2.5. To abbreviate some formulas, in the remainder of this paper $Q$ is the constant $Q:=N+2^{\alpha+1 / q} /|1-q \alpha|^{1 / q}$.

We can now establish our first result on the existence of a mild solution.

Theorem 2.6. Assume that $H_{1}, H_{4}$ are satisfied and there are $r>0$ and $\Theta \in(0,1)$ such that

$$
\begin{aligned}
\Lambda= & C_{\alpha}\left(\frac{a}{1-\alpha}\|f(\cdot, 0)\|_{C([0, a] ; X)}+\sum_{i=1}^{N}\left\|I_{i}(0)\right\|\right)<(1-\Theta) r, \\
& C_{\alpha}\left(\left\|\mathcal{K}_{f}\right\|_{L^{q^{\prime}}([0, a])} Q \frac{2^{\alpha} a^{1 / q-\alpha}}{|1-q \alpha|^{1 / q}} r+\sum_{i=1}^{N} \frac{\mathcal{K}_{I_{i}}\left(\frac{r}{\delta_{i-1}^{\alpha}}\right)}{\delta_{i-1}^{\alpha}}\right) \leq \Theta r .
\end{aligned}
$$

If $\left\|x_{0}\right\|<(r(1-\Theta)-\Lambda) / C_{\alpha}$, then there exists a unique mild solution $u \in \mathcal{P} C_{\alpha}\left(X_{\alpha}\right)$ of (1.1)-(1.3).

Proof. Let $\Gamma: \mathcal{P} C_{\alpha}\left(X_{\alpha}\right) \rightarrow \mathcal{P C} C_{\alpha}\left(X_{\alpha}\right)$ be the map defined by

$$
\Gamma u(t)=T(t) x_{0}+\int_{0}^{t} T(t-s) f(s, u(s)) d s+\sum_{t_{i}<t} T\left(t-t_{i}\right) I_{i}\left(u\left(t_{i}\right)\right), \quad t \in(0, a] .
$$

Next, we prove that the map $\Gamma$ is a contraction on $B_{r}\left(0, \mathcal{P} C_{\alpha}\left(X_{\alpha}\right)\right)$. To begin, we show that $\Gamma$ has values in $B_{r}\left(0, \mathcal{P} C_{\alpha}\left(X_{\alpha}\right)\right)$. Let $u \in B_{r}\left(0, \mathcal{P} C_{\alpha}\left(X_{\alpha}\right)\right)$. By using Lemma 2.4, 
for $t \in\left(t_{j}, t_{j+1}\right]$ we have that

$$
\begin{aligned}
\left\|(-A)^{\alpha} \Gamma u(t)\right\| \leq & \frac{C_{\alpha}}{t^{\alpha}}\left\|x_{0}\right\|+C_{\alpha} \int_{0}^{t} \frac{\mathcal{K}_{f}(s)\|u\|_{\alpha}}{(t-s)^{\alpha}\left(s-t_{i}\right)^{\alpha}} d s+C_{\alpha} \int_{0}^{t} \frac{\|f(s, 0)\|}{(t-s)^{\alpha}} d s \\
& +C_{\alpha} \sum_{i=1}^{j}\left(\frac{\mathcal{K}_{I_{i}}\left(\frac{r}{\delta_{i-1}^{\alpha}}\right)}{\left(t-t_{i}\right)^{\alpha} \delta_{i-1}^{\alpha}}\|u\|_{\alpha}^{q}+\frac{\left\|I_{i}(0)\right\|}{\left(t-t_{i}\right)^{\alpha}}\right) \\
\leq & \frac{C_{\alpha}\left\|x_{0}\right\|}{\left(t-t_{j}\right)^{\alpha}}+\frac{C_{\alpha}\left\|\mathcal{K}_{f}\right\|_{L^{q^{\prime}}\left(\left[0, t_{j}\right]\right)}}{\left(t-t_{j}\right)^{\alpha}} Q\left(\int_{0}^{a} 2^{q \alpha} \frac{\|u\|_{\alpha}}{s^{q \alpha}} d s\right)^{1 / q} \\
& +\frac{C_{\alpha}\left(t-t_{j}\right)^{\alpha} t^{1-\alpha}}{\left(t-t_{j}\right)^{\alpha}(1-\alpha)}\|f(\cdot, 0)\|_{C([0, a] ; X)} \\
& +\frac{C_{\alpha}}{\left(t-t_{j}\right)^{\alpha}} \sum_{i=1}^{j}\left(\frac{\mathcal{K}_{I_{i}}\left(\frac{r}{\delta_{i-1}^{\alpha}}\right)}{\delta_{i-1}^{\alpha}}\|u\|_{\alpha}+\left\|I_{i}(0)\right\|\right)
\end{aligned}
$$

so that

$$
\begin{aligned}
\left\|\Gamma_{n} u\right\|_{\alpha, j} \leq C_{\alpha}\left(\left\|x_{0}\right\|+\left\|\mathcal{K}_{f}\right\|_{\left.L^{q^{\prime}\left(\left[0, t_{j}\right]\right.}\right)} Q \frac{2^{\alpha} a^{1 / q-\alpha}}{|1-q \alpha|^{1 / q}}\|u\|_{\alpha}\right) \\
+\frac{C_{\alpha} a}{1-\alpha}\|f(\cdot, 0)\|_{C([0, a] ; X)}+C_{\alpha} \sum_{i=1}^{j}\left(\frac{\mathcal{K}_{I_{i}}\left(\frac{r}{\delta_{i-1}^{\alpha}}\right)}{\delta_{i-1}^{\alpha}}\|u\|_{\alpha}+\left\|I_{i}(0)\right\|\right),
\end{aligned}
$$

from which

$$
\|\Gamma u\|_{\alpha} \leq C_{\alpha}\left\|x_{0}\right\|+\Theta r+C_{\alpha}\left(\frac{a}{1-\alpha}\|f(\cdot, 0)\|_{C([0, a] ; X)}+\sum_{i=1}^{N}\left\|I_{i}(0)\right\|\right) \leq r
$$

and $\Gamma u \in B_{r}\left(0, \mathcal{P} C_{\alpha}\left(X_{\alpha}\right)\right)$. Moreover, arguing as above, for $u, v \in B_{r}\left(0, \mathcal{P} C_{\alpha}\left(X_{\alpha}\right)\right)$,

$$
\|\Gamma u-\Gamma v\|_{\alpha, j} \leq C_{\alpha}\left(\left\|\mathcal{K}_{f}\right\|_{L^{q^{\prime}}([0, a])} Q \frac{2^{\alpha} a^{1 / q-\alpha}}{|1-q \alpha|^{1 / q}}+\sum_{i=1}^{N} \frac{\mathcal{K}_{I_{i}}\left(\frac{r}{\delta_{i-1}^{\alpha}}\right)}{\delta_{i-1}^{\alpha}}\right)\|u-v\|_{\alpha},
$$

which implies that $\|\Gamma u-\Gamma v\|_{\alpha} \leq \Theta\|u-v\|_{\alpha}$. Thus, $\Gamma$ is a contraction and there exists a unique fixed point $v \in B_{r}\left(0, \mathcal{P} C_{\alpha}\left(X_{\alpha}\right)\right)$ of $\Gamma(\cdot)$.

Let $u:[0, a] \rightarrow X$ be defined by $u(t)=v(t)$ for $t \neq 0$ and $u(0)=x_{0}$. To complete the proof, next we prove that $u(\cdot)$ is a mild solution of (1.1)-(1.3). It is easy to see that $u(\cdot)$ is left continuous on $[0, a]$ and that all the limits $u\left(t_{i}^{+}\right)$exist. Thus, it remains to show that $\|\Gamma u\|_{\mathcal{P}(X)}$ is finite. To prove this, we note that for $s \in\left(t_{i}, t_{i+1}\right]$,

$$
\begin{aligned}
\int_{t_{i}}^{s}\|f(\tau, u(\tau))\| d \tau & \leq \int_{t_{i}}^{s} \frac{\mathcal{K}_{f}(\tau)\|u\|_{\alpha}}{\left(\tau-t_{i}\right)^{\alpha}} d \tau+\int_{t_{i}}^{s}\|f(\tau, 0)\| d \tau \\
& \leq \Upsilon:=\left\|\mathcal{K}_{f}\right\|_{L^{q^{\prime}([0, a])}} \frac{r a^{1 / q-\alpha}}{(1-q \alpha)^{1 / q}}+\|f(\cdot, 0)\|_{L^{1}([0, a])} .
\end{aligned}
$$


By using condition (2.4) and inequality (2.5), for $t \in\left(t_{j}, t_{j+1}\right]$ we see that

$$
\begin{gathered}
\|u(t)\| \leq C_{0}\left\|x_{0}\right\|+C_{0} \sum_{i=0}^{j-1} \int_{t_{i}}^{t_{i+1}}\|f(\tau, u(\tau))\| d \tau+C_{0} \int_{t_{j}}^{t}\|f(\tau, u(\tau))\| d \tau \\
\quad+C_{0} \sum_{i=1}^{j} \frac{\mathcal{K}_{I_{i}\left(\frac{r}{\delta_{i-1}^{\alpha}}\right) r}}{\delta_{i-1}^{\alpha}}+C_{0} \sum_{i=1}^{j}\left\|I_{i}(0)\right\| \\
\leq C_{0}\left\|x_{0}\right\|+C_{0} N \Upsilon+\frac{C_{0}}{C_{\alpha}} r+C_{0} \sum_{i=1}^{j}\left\|I_{i}(0)\right\|,
\end{gathered}
$$

which implies that $\|u\|_{\mathcal{P} C(X)}<\infty$. The proof is complete.

Next, we establish some consequences of Theorem 2.6.

Corollary 2.7. If the conditions $\mathrm{H}_{1}, \mathrm{H}_{4}$ hold and

$$
C_{\alpha}\left(Q \frac{2^{\alpha} a^{1 / q-\alpha}}{|1-q \alpha|^{1 / q}}\left\|\mathcal{K}_{f}\right\|_{L^{q^{\prime}([0, a])}}+\limsup _{l \rightarrow \infty} \frac{1}{l} \sum_{i=1}^{N} \frac{\mathcal{K}_{I_{i}}\left(\frac{l}{\delta_{i-1}^{\alpha}}\right)}{\delta_{i-1}^{\alpha}}\right)<1,
$$

then there exists a unique mild solution $u \in \mathcal{P} C_{\alpha}\left(X_{\alpha}\right)$ of (1.1)-(1.3). In particular, if the functions $\mathcal{K}_{f}(\cdot), \mathcal{K}_{I_{i}}(\cdot)$ are bounded and $C_{\alpha} Q\left(2^{\alpha} a^{1-\alpha} /|1-q \alpha|^{1 / q}\right)\left\|\mathcal{K}_{f}\right\|_{L^{\infty}([0, a])}<1$, then there exists a unique mild solution $u \in \mathcal{P} C_{\alpha}\left(X_{\alpha}\right)$ for (1.1)-(1.3).

Proof. Let $\Lambda$ be the number introduced in (2.3). From the assumption, we can select numbers $r>0$ and $\Theta \in(0,1)$ such that

$$
C_{\alpha}\left(Q \frac{2^{\alpha} a^{1 / q-\alpha}}{|1-q \alpha|^{1 / q}}\left\|\mathcal{K}_{f}\right\|_{L^{q^{\prime}([0, a])}} r+\sum_{i=1}^{N} \frac{\mathcal{K}_{I_{i}}\left(\frac{r}{\delta_{i-1}^{\alpha}}\right)}{\delta_{i-1}^{\alpha}}\right)<\Theta r,
$$

$\Lambda<(1-\Theta) r$ and $\left\|x_{0}\right\|<(r(1-\Theta)-\Lambda) / C_{\alpha}$. Now, the assertion follows from Theorem 2.6.

REMARK 2.8. It is convenient to include some comments on Theorem 2.6. The assumption on the existence of $r$ is a restriction, since under this condition the result is only applicable for a initial condition in a neighbourhood of zero. However, this restriction is natural and predicable for two reasons.

(a) Our abstract approach is designed to study partial differential equations with impulsive conditions involving nonlinear expressions of the solution. Note that if some of the functions $I_{i}$ are nonlinear and $\lim _{s \rightarrow \infty} \mathcal{K}_{I_{i}}(s) s^{-\gamma}=\infty$ for some $\gamma>1$ (respectively, $\lim _{s \rightarrow 0} \mathcal{K}_{I_{i}}(s) s^{-\gamma}=\infty$ for some $\gamma<1$ ), then (2.4) is not satisfied for $r$ large (respectively, small).

(b) To preserve the basic characteristic of the problem (the presence of impulses), we need to establish the existence of a mild solution on an interval containing the times $t_{i}$. In problems without impulses, the dependence on $r$ can be eliminated by studying the existence of solutions local in time. 
To prove our main result (Theorem 2.13), we consider an approximation scheme based on the solvability of the associated abstract integral equations

$$
u(t)=T(t) x_{0}+\int_{0}^{t} T(t-s) f(s, u(s)) d s+T\left(\frac{1}{n}\right) \sum_{t_{i}<t} T\left(t-t_{i}\right) I_{i}\left(u\left(t_{i}\right)\right), \quad n \in \mathbb{N} .
$$

To begin, we study the existence of solutions for the integral equation (2.6).

Theorem 2.9. Assume that $H_{2}$ and $H_{3}$ are satisfied, $(T(t))_{t \geq 0}$ is compact, the function $\tau \rightarrow W_{f}^{q}\left(1 / \tau^{\alpha}\right)$ is integrable on $[0, a]$,

$$
\lim _{h \downarrow 0}\left\|m_{f}\right\|_{L^{q^{\prime}([c, c+h])}} \frac{1}{h^{\alpha}}\left(\int_{0}^{h} W_{f}^{q}\left(\frac{1}{s^{\alpha}}\right) d s\right)^{1 / q}=0
$$

for all $c \in[0, a), \lim _{h \rightarrow 0}\left\|m_{f}\right\|_{L^{q^{\prime}([d-h, d])}}\left(1 / h^{\alpha}\right)\left(\int_{0}^{h} W_{f}^{q}\left(1 / s^{\alpha}\right) d s\right)^{1 / q}=0$ uniformly for $d$ in compact subsets of $(0, a]$, and there is $r>0$ such that

$$
C_{\alpha}\left(\left\|x_{0}\right\|+Q\left\|m_{f}\right\|_{L^{q^{\prime}}([0, a])}\left(\int_{0}^{a} W_{f}^{q}\left(\frac{2^{\alpha} r}{s^{\alpha}}\right) d s\right)^{1 / q}+C_{0} \sum_{i=1}^{N}\left(\frac{c_{i}\left(\frac{r}{\delta_{i-1}^{\alpha}}\right) r}{\delta_{i-1}^{\alpha}}+d_{i}\right)\right) \leq r .
$$

Then, for all $n \in \mathbb{N}$, there exists a solution $u_{n} \in B_{r}\left(0, \mathcal{P} C_{\alpha}\left(X_{\alpha}\right)\right) \cap \mathcal{P C}(X)$ of (2.6).

Proof. For $n \in \mathbb{N}$, we define the $\operatorname{map} \Gamma_{n}: \mathcal{P} C_{\alpha}\left(X_{\alpha}\right) \rightarrow \mathcal{P} C_{\alpha}\left(X_{\alpha}\right)$ by

$$
\Gamma_{n} u(t)=T(t) x_{0}+\int_{0}^{t} T(t-s) f(s, u(s)) d s+T\left(\frac{1}{n}\right) \sum_{t_{i}<t} T\left(t-t_{i}\right) I_{i}\left(u\left(t_{i}\right)\right) .
$$

Next, we prove that $\Gamma_{n}$ is a condensing map on $B_{r}\left(0, \mathcal{P} C_{\alpha}\left(X_{\alpha}\right)\right)$. To begin, we show that $\Gamma$ has values in $B_{r}\left(0, \mathcal{P} C_{\alpha}\left(X_{\alpha}\right)\right)$. Let $u \in B_{r}\left(0, \mathcal{P} C_{\alpha}\left(X_{\alpha}\right)\right)$. From Lemma 2.4, for $t \in\left(t_{j}, t_{j+1}\right]$,

$$
\begin{gathered}
\left\|(-A)^{\alpha} \Gamma_{n} u(t)\right\| \leq \frac{C_{\alpha}}{t^{\alpha}}\left\|x_{0}\right\|+C_{\alpha} \int_{0}^{t} \frac{m_{f}(s)}{(t-s)^{\alpha}} W_{f}\left(\left\|(-A)^{\alpha} u(s)\right\|\right) d s \\
\quad+\sum_{i=1}^{j} \frac{C_{\alpha} C_{0}}{\left(t-t_{i}\right)^{\alpha}}\left(\frac{c_{i}\left(\frac{r}{\delta_{i-1}^{\alpha}}\right) r}{\delta_{i-1}^{\alpha}}+d_{i}\right) \\
\leq \frac{C_{\alpha}\left\|x_{0}\right\|}{\left(t-t_{j}\right)^{\alpha}}+\frac{\left\|m_{f}\right\|_{L^{q^{\prime}}([0, a])}}{\left(t-t_{j}\right)^{\alpha}} Q\left(\int_{0}^{a} W_{f}\left(\frac{2^{\alpha} r}{s^{\alpha}}\right) d s\right)^{1 / q} \\
\quad+\frac{C_{\alpha} C_{0}}{\left(t-t_{j}\right)^{\alpha}} \sum_{i=1}^{j}\left(\frac{c_{i}\left(\frac{r}{\delta_{i-1}^{\alpha}}\right) r}{\delta_{i-1}^{\alpha}}+d_{i}\right),
\end{gathered}
$$

and hence,

$$
\begin{gathered}
\left\|\Gamma_{n} u\right\|_{\alpha, j} \leq C_{\alpha}\left(\left\|x_{0}\right\|+\left\|m_{f}\right\|_{L^{q^{\prime}([0, a])}} Q\left(\int_{0}^{a} W_{f}\left(\frac{2^{\alpha} r}{s^{\alpha}}\right) d s\right)^{1 / q}\right) \\
+C_{\alpha} C_{0} \sum_{i=1}^{j}\left(\frac{c_{i}\left(\frac{r}{\delta_{i-1}^{\alpha}}\right) r}{\delta_{i-1}^{\alpha}}+d_{i}\right),
\end{gathered}
$$

which implies that $\left\|\Gamma_{n} u\right\|_{\alpha} \leq r$ and $\Gamma_{n} B_{r}\left(0, \mathcal{P} C_{\alpha}\left(X_{\alpha}\right)\right) \subset B_{r}\left(0, \mathcal{P} C_{\alpha}\left(X_{\alpha}\right)\right)$. 
To show that the map $\Gamma_{n}$ is completely continuous, we introduce the decomposition $\Gamma_{n}=\Gamma^{1}+\Gamma^{2}+\Gamma_{n}^{3}$ where $\Gamma^{1} u(t)=T(t) x_{0}, \Gamma^{2} u(t)=\int_{0}^{t} T(t-s) f(s, u(s)) d s$ and $\Gamma_{n}^{3} u(t)=$ $T(1 / n) \sum_{t_{i}<t} T\left(t-t_{i}\right) I_{i}\left(u\left(t_{i}\right)\right)$. The continuity of the map $\Gamma_{n}(\cdot)$ is proved by a standard procedure. We divide the remainder of the proof into several steps. Next, we use the notation $\Gamma^{i} B_{r}\left(0, \mathcal{P} C_{\alpha}\left(X_{\alpha}\right)\right)(s)=\left\{\Gamma^{i} u(s): u \in B_{r}\left(0, \mathcal{P} C_{\alpha}\left(X_{\alpha}\right)\right)\right\}$.

Step 1. For $t_{j}<c<d \leq t_{j+1}$, the set $\cup_{s \in[c, d]} \Gamma^{2} B_{r}\left(0, \mathcal{P} C_{\alpha}\left(X_{\alpha}\right)\right)(s)$ is relatively compact in $X_{\alpha}$.

Let $t_{j}<\mu<c$. From Lemmas 2.3 and 2.4, for $t \in[c, d], u \in B_{r}\left(0, \mathcal{P} C_{\alpha}\left(X_{\alpha}\right)\right)$ and $0<\varepsilon<(c-\mu) / 2$,

$$
\begin{aligned}
& \left\|(-A)^{\alpha} \Gamma^{2} u(t)\right\| \leq C_{\alpha} \int_{0}^{t} \frac{m_{f}(s)}{(t-s)^{\alpha}} W_{f}\left(\left\|(-A)^{\alpha} u(s)\right\|\right) d s \\
& \quad \leq r_{1}:=\frac{C_{\alpha}\left\|m_{f}\right\|_{L^{\prime}([0, a])}}{\left(\mu-t_{j}\right)^{\alpha}} Q\left(\int_{0}^{a} W_{f}\left(\frac{2^{\alpha} r}{\tau^{\alpha}}\right) d \tau\right)^{1 / q} \\
& \left\|\int_{t-\varepsilon}^{t}(-A)^{\alpha} T(t-\tau) f(\tau, u(\tau)) d s\right\| \\
& \quad \leq C_{\alpha} \int_{0}^{t} \frac{m_{f}(s)}{(t-s)^{\alpha}} W_{f}\left(\left\|(-A)^{\alpha} u(s)\right\|\right) d s \\
& \quad \leq \frac{C_{\alpha} 2^{\alpha+1 / q}}{|1-q \alpha|^{1 / q}}\left\|m_{f}\right\|_{L^{q^{\prime}}([-\varepsilon, t])} \frac{1}{\varepsilon^{\alpha}}\left(\int_{0}^{\varepsilon / 2} W_{f}^{q}\left(\frac{2^{\alpha} r}{\tau^{\alpha}}\right) d \tau\right)^{1 / q} \\
& \quad \leq r_{\varepsilon}:=\frac{C_{\alpha} 2^{\alpha+1 / q}}{|1-q \alpha|^{1 / q}} \sup _{s \in[\mu, d]}\left\|m_{f}\right\|_{L^{q^{\prime}}([s-\varepsilon, s])} \frac{1}{\varepsilon^{\alpha}}\left(\int_{0}^{\varepsilon} W_{f}^{q}\left(\frac{2^{\alpha} r}{\tau^{\alpha}}\right) d \tau\right)^{1 / q} .
\end{aligned}
$$

By noting that the above estimates are independent of $t \in[c, d]$, from the decomposition

$$
\begin{aligned}
(-A)^{\alpha} \Gamma^{2} u(s)= & T(\varepsilon) \int_{0}^{s-\varepsilon}(-A)^{\alpha} T(s-\varepsilon-\tau) f(\tau, u(\tau)) d \tau \\
& +\int_{s-\varepsilon}^{s}(-A)^{\alpha} T(s-\tau) f(\tau, u(\tau)) d \tau
\end{aligned}
$$

we infer that $\cup_{s \in[c, d]}(-A)^{\alpha} \Gamma^{2} B_{r}\left(0, \mathcal{P} C_{\alpha}\left(X_{\alpha}\right)\right)(s) \subset T(\varepsilon) B_{r_{1}}(0, X)+B_{2 r_{\varepsilon}}(0, X)$, which proves the assertion since $T(\varepsilon) B_{r_{1}}(0, X)$ is compact in $X$ and $r_{\varepsilon} \rightarrow 0$ as $\varepsilon \rightarrow 0$.

Step 2. $\Gamma^{2} B_{r}\left(0, \mathcal{P} C_{\alpha}\left(X_{\alpha}\right)\right)=\left\{\Gamma^{2} u: u \in B_{r}\left(0, \mathcal{P} C_{\alpha}\left(X_{\alpha}\right)\right)\right\} \quad$ is an equicontinuous subset of $C\left([0, a] ; X_{\alpha}\right)$.

Let $t \in(0, a]$. To begin, we assume that $t \in\left(t_{j}, t_{j+1}\right)$. Since $T(\cdot) \in C((0, a], \mathcal{L}(X))$ and $\Gamma^{2} B_{r}\left(0, \mathcal{P} C_{\alpha}\left(X_{\alpha}\right)\right)(t)$ is relatively compact in $X$, for given $\varepsilon>0$ we can select $0<\delta \leq \min \left\{t_{j+1}-t\right\}$ such that $\|(T(\theta)-I) x\| \leq \varepsilon$ for every $x \in \Gamma^{2} B_{r}\left(0, \mathcal{P} C_{\alpha}\left(X_{\alpha}\right)\right)(t)$ and 
all $0<\theta \leq \delta$. Then, for $0<h<\delta$ and $u \in B_{r}\left(0, \mathcal{P} C_{\alpha}\left(X_{\alpha}\right)\right)$,

$$
\begin{aligned}
&\left\|(-A)^{\alpha} \Gamma^{2} u(t+h)-(-A)^{\alpha} \Gamma^{2} u(t)\right\| \\
& \leq\left\|(T(h)-I)(-A)^{\alpha} \Gamma^{2} u(t)\right\|+\int_{t}^{t+h}\left\|(-A)^{\alpha} T(t+h-s) f(s, u(s))\right\| d s \\
& \leq \sup \left\{\left\|(T(h)-I)(-A)^{\alpha} \Gamma^{2} v(t)\right\|: v \in B_{r}\left(0, \mathcal{P} C_{\alpha}\left(X_{\alpha}\right)\right)\right\} \\
&+C_{\alpha} \int_{t}^{t+h} \frac{m_{f}(s)}{(t+h-s)^{\alpha}} W_{f}\left(\frac{\|u\|_{\alpha}}{(s-t)^{\alpha}}\right) d s \\
& \leq \varepsilon+\frac{C_{\alpha} 2^{\alpha+1 / q}\left\|m_{f}\right\|_{L^{q^{\prime}}([t, t+h])}}{|1-q \alpha|^{1 / q}} \frac{1}{h^{\alpha}}\left(\int_{0}^{h / 2} W_{f}^{q}\left(\frac{2^{\alpha} r}{s^{\alpha}}\right) d s\right)^{1 / q},
\end{aligned}
$$

which shows that $(-A)^{\alpha} \Gamma^{2} B_{r}\left(0, \mathcal{P} C_{\alpha}\left(X_{\alpha}\right)\right)$ is right equicontinuous at $t$.

We now show the left equicontinuity for the case where $t \in\left(t_{j}, t_{j+1}\right]$. Let $t_{j}<$ $\mu<c<t$. By noting that the set $\cup_{s \in[\mu, t]} \Gamma^{2} B_{r}\left(0, \mathcal{P} C_{\alpha}\left(X_{\alpha}\right)\right)(s)$ is relatively compact in $X_{\alpha}$ (see Step 1), for $\varepsilon>0$ there exists $0<\delta<(c-\mu) / 2$ such that $\|(T(\theta)-I) x\| \leq \varepsilon$ for every $x \in \cup_{s \in[\mu, t]} \Gamma^{2} B_{r}\left(0, \mathcal{P} C_{\alpha}\left(X_{\alpha}\right)\right)(s)$ and all $0<\theta \leq \delta$. Then, for $0<h<\delta$ and $u \in B_{r}\left(0, \mathcal{P} C_{\alpha}\left(X_{\alpha}\right)\right)$,

$$
\begin{aligned}
\|( & -A)^{\alpha} \Gamma^{2} u(t-h)-(-A)^{\alpha} \Gamma^{2} u(t) \| \\
\leq & \left\|(I-T(h))(-A)^{\alpha} \Gamma^{2} u(t-h)\right\|+\int_{t-h}^{t}\left\|(-A)^{\alpha} T(t-s) f(s, u(s))\right\| d s \\
\leq & \sup \left\{\left\|(I-T(h))(-A)^{\alpha} \Gamma^{2} v(s)\right\|: s \in[\mu, t], v \in B_{r}\left(0, \mathcal{P} C_{\alpha}\left(X_{\alpha}\right)\right)\right\} \\
& +C_{\alpha} \int_{t-h}^{t} \frac{m_{f}(s)}{(t-s)^{\alpha}} W_{f}\left(\frac{\|u\|_{\alpha}}{(s-(t-h))^{\alpha}}\right) d s \\
\leq & +\frac{C_{\alpha} 2^{\alpha+1 / q}}{(1-q \alpha)^{1 / q}}\left\|m_{f}\right\|_{L^{q^{\prime}}([t-h, t] ; \mathbb{R})} \frac{1}{h^{\alpha}}\left(\int_{0}^{h} W_{f}^{q}\left(\frac{2^{\alpha} r}{s^{\alpha}}\right) d s\right)^{1 / q},
\end{aligned}
$$

which shows the left equicontinuity at $t$.

Step 3. For $t_{j}<c<d \leq t_{j+1}$, the set $\cup_{s \in[c, d]} \Gamma_{n}^{3} B_{r}\left(0, \mathcal{P} C_{\alpha}\left(X_{\alpha}\right)\right)(s)$ is relatively compact in $X_{\alpha}$.

The case where $j=0$ is obvious. Assume that $j \geq 1$ and let $t_{j}<\mu<c$. For $u \in B_{r}\left(0, \mathcal{P} C_{\alpha}\left(X_{\alpha}\right)\right)$ and $s \in[\mu, d]$ we see that

$$
\left\|(-A)^{\alpha} \Gamma_{n}^{3} u(s)\right\| \leq r^{*}:=\frac{C_{\alpha} C_{0}}{\left(\mu-t_{j}\right)^{\alpha}} \sum_{i=1}^{j}\left(\frac{c_{i}\left(\frac{r}{\delta_{i-1}^{\alpha}}\right) r}{\delta_{i-1}^{\alpha}}+d_{i}\right),
$$

and hence, $\cup_{\tau \in[\mu, d]}(-A)^{\alpha} \Gamma_{n}^{3} B_{r}\left(0, \mathcal{P} C_{\alpha}\left(X_{\alpha}\right)\right)(\tau) \subset B_{r^{*}}(0, X)$. Thus, for $t \in[c, d], u \in$ $B_{r}\left(0, \mathcal{P} C_{\alpha}\left(X_{\alpha}\right)\right)$ and $0<\varepsilon<(c-\mu) / 2$,

$$
(-A)^{\alpha} \Gamma_{n}^{3} u(t)=T(\varepsilon)(-A)^{\alpha} \Gamma_{n}^{3} u(t-\varepsilon) \subset T(\varepsilon) B_{r^{*}}(0, X),
$$

from which we infer that $\cup_{s \in[c, d]}(-A)^{\alpha} \Gamma_{n}^{3} B_{r}\left(0, \mathcal{P} C_{\alpha}\left(X_{\alpha}\right)\right)(s) \subset T(\varepsilon) B_{r^{*}}(0, X)$. This proves the assertion since $T(\varepsilon)$ is compact. 
Step 4. The set of functions $\Gamma_{n}^{3} B_{r}\left(0, \mathcal{P} C_{\alpha}\left(X_{\alpha}\right)\right)=\left\{\Gamma_{n}^{3} u: u \in B_{r}\left(0, \mathcal{P} C_{\alpha}\left(X_{\alpha}\right)\right)\right\}$ is equicontinuous at $t \neq t_{i}$ and left equicontinuous $t=t_{i}$ in the norm of $X_{\alpha}$.

The case $t \in\left(0, t_{1}\right]$ is trivial. Assume that $t \in\left(t_{j}, t_{j+1}\right)$ for some $j \geq 1$. By using the fact that $(-A)^{\alpha} \Gamma_{n}^{3} B_{r}\left(0, \mathcal{P} C_{\alpha}\left(X_{\alpha}\right)\right)(t)$ is relatively compact in $X$, for $\varepsilon>0$ we select $0<\delta<t_{j+1}-t$ such that $\|(T(\theta)-I) x\| \leq \varepsilon$ for all $x \in(-A)^{\alpha} \Gamma_{n}^{3} B_{r}\left(0, \mathcal{P} C_{\alpha}\left(X_{\alpha}\right)\right)(t)$ and every $0<\theta \leq \delta$. Then, for $u \in B_{r}\left(0, \mathcal{P} C_{\alpha}\left(X_{\alpha}\right)\right)$ and $0<h<\delta$,

$$
\begin{aligned}
& \left\|(-A)^{\alpha} \Gamma_{n}^{3} u(t+h)-(-A)^{\alpha} \Gamma_{n}^{3} u(t)\right\|=\left\|(T(h)-I)(-A)^{\alpha} \Gamma_{n}^{3} u(t)\right\| \\
& \quad \leq \sup \left\{(T(h)-I)(-A)^{\alpha} \Gamma_{n}^{3} v(s) \|: v \in B_{r}\left(0, \mathcal{P} C_{\alpha}\left(X_{\alpha}\right)\right)\right\} \\
& \quad \leq \varepsilon
\end{aligned}
$$

which shows that $(-A)^{\alpha} \Gamma_{n}^{3} B_{r}\left(0, \mathcal{P} C_{\alpha}\left(X_{\alpha}\right)\right)$ is right equicontinuous at $t$.

We now prove left equicontinuity for the case where $t \in\left(t_{j}, t_{j+1}\right]$. Let $t_{j}<$ $\mu<c<t$. Since the set $\cup_{s \in[\mu, t]}(-A)^{\alpha} \Gamma_{n}^{3} B_{r}\left(0, \mathcal{P} C_{\alpha}\left(X_{\alpha}\right)\right)(s)$ is relatively compact in $X$, for $\varepsilon>0$ we choose $0<\delta<(c-\mu) / 2$ such that $\|(T(\theta)-I) x\| \leq \varepsilon$ for all $x \in$ $\cup_{s \in[\mu, t]}(-A)^{\alpha} \Gamma_{n}^{3} B_{r}\left(0, \mathcal{P} C_{\alpha}\left(X_{\alpha}\right)\right)(s)$ and each $0<\theta<\delta$. Under these conditions, for $u \in B_{r}\left(0, \mathcal{P} C_{\alpha}\left(X_{\alpha}\right)\right)$ and $0<h<\delta$,

$$
\begin{aligned}
& \left\|(-A)^{\alpha} \Gamma_{n}^{3} u(t-h)-(-A)^{\alpha} \Gamma_{n}^{3} u(t)\right\|=\left\|(I-T(h))(-A)^{\alpha} \Gamma_{n}^{3} u(t-h)\right\| \\
& \quad \leq \sup \left\{\left\|(I-T(h))(-A)^{\alpha} \Gamma_{n}^{3} v(s)\right\|: s \in[\mu, t], v \in B_{r}\left(0, \mathcal{P} C_{\alpha}\left(X_{\alpha}\right)\right)\right\} \\
& \quad \leq \varepsilon
\end{aligned}
$$

which proves the left equicontinuity at $t$. This completes the proof that $\Gamma_{n}^{3} B_{r}\left(0, \mathcal{P} C_{\alpha}\left(X_{\alpha}\right)\right)$ is equicontinuous at $t \neq t_{i}$.

Step 5. For every $j \in\{1, \ldots, N\}, \lim _{t \downarrow t_{j}}\left(t-t_{j}\right)^{\alpha}(-A)^{\alpha} \Gamma_{n}^{3} u(t)=0$ uniformly for $u \in$ $B_{r}\left(0, \mathcal{P} C_{\alpha}\left(X_{\alpha}\right)\right)$.

For $u \in B_{r}\left(0, \mathcal{P} C_{\alpha}\left(X_{\alpha}\right)\right)$ and $t \in\left(t_{j}, t_{j+1}\right)$,

$$
\left(t-t_{j}\right)^{\alpha}\left\|(-A)^{\alpha} \Gamma_{n}^{3} u(t)\right\| \leq \frac{\left(t-t_{j}\right)^{\alpha}}{\left(t-t_{j}+\frac{1}{n}\right)^{\alpha}} C_{\alpha} \sum_{i=1}^{N}\left(\frac{c_{i}\left(\frac{r}{\delta_{i-1}^{\alpha}}\right) r}{\delta_{i-1}^{\alpha}}+d_{i}\right),
$$

which proves the assertion since $\left(t-t_{j}\right)^{\alpha} /\left(t-t_{j}+1 / n\right)^{\alpha} \rightarrow 0$ as $t \downarrow t_{j}$.

From Steps 1-5 and Lemma 1.2 it follows that $\Gamma_{n}$ is completely continuous from $B_{r}\left(0, \mathcal{P} C_{\alpha}\left(X_{\alpha}\right)\right)$ into $B_{r}\left(0, \mathcal{P} C_{\alpha}\left(X_{\alpha}\right)\right)$ and from Schauder's fixed point criteria there exists a fixed point $v_{n}$ of $\Gamma_{n}$. Let $u_{n}:[0, a] \rightarrow X$ be defined by $u_{n}(0)=x_{0}$ and $u_{n}(t)=v_{n}(t)$ for $t \in(0, a]$. It is easy to see that $u_{n}(\cdot)$ is a solution of the integral equation (2.6) and $u(\cdot)$ is continuous at zero. In order to prove that $u_{n} \in \mathcal{P} C(X)$, we note that

$$
\int_{t_{i}}^{t_{i+1}}\left\|f\left(\tau, u_{n}(\tau)\right)\right\| d \tau \leq \mathfrak{Q}:=\left\|m_{f}\right\|_{L^{q^{\prime}([0, a])}}\left(\int_{0}^{a} W_{f}^{q}\left(\frac{r}{\tau^{\alpha}}\right) d \tau\right)^{1 / q},
$$


for all $i \in\{0, \ldots, N\}$. Using (2.10) and (2.7), for $t \in\left(t_{j}, t_{j+1}\right]$ it follows that

$$
\begin{aligned}
\left\|u_{n}(t)\right\| & \leq C_{0}\left\|x_{0}\right\|+C_{0} \sum_{i=0}^{j} \int_{t_{i}}^{t_{i+1}}\left\|f\left(\tau, u_{n}(\tau)\right)\right\| d \tau+C_{0}^{2} \sum_{i=1}^{j}\left(\frac{c_{i}\left(\frac{r}{\delta_{i-1}^{\alpha}}\right) r}{\delta_{i-1}^{\alpha}}+d_{i}\right) \\
& \leq C_{0}\left\|x_{0}\right\|+C_{0} N \mathfrak{Q}+\frac{C_{0}}{C_{\alpha}} r,
\end{aligned}
$$

which implies that $\left\|u_{n}\right\|_{\mathcal{P} C(X)} \leq C_{0}\left(\left\|x_{0}\right\|+N \mathfrak{Q}+C_{0} r / C_{\alpha}\right)$ and $u_{n} \in \mathcal{P} C(X)$. This completes the proof.

REMARK 2.10. In the remainder of this section, we always assume that the assumptions in Theorem 2.9 are fulfilled. Next, for $n \in \mathbb{N}$ we use the notation $u_{n}$ to represent a given solution of the integral equation (2.6). For convenience, we rewrite the functions $u_{n}$ in the form $u_{n}=\sum_{i=1}^{3} u_{n}^{i}$ where $u_{n}^{1}(t)=T(t) x_{0}, u_{n}^{2}(t)=\int_{0}^{t} T(t-s) f\left(s, u_{n}(s)\right) d s$ and $u_{n}^{3}(t)=T(1 / n) \sum_{t_{i}<t} T\left(t-t_{i}\right) I_{i}\left(u_{n}\left(t_{i}\right)\right)$.

We now consider some properties of the set $\left\{u_{n}: n \in \mathbb{N}\right\}$. The proof of Lemma 2.11 can be deduced from the proof of Theorem 2.9. We include it for completeness.

Lemma 2.11. If the assumptions in Theorem 2.9 are satisfied, then

(a) the set $\left\{u_{n}^{i}: i=1,2,3, n \in \mathbb{N}\right\}$ is bounded in $\mathcal{P} C(X)$ and $\left\{u_{n}^{i}: i=1,2,3, n \in \mathbb{N}\right\} \subset$ $B_{r}\left(0, \mathcal{P} C_{\alpha}\left(X_{\alpha}\right)\right)$,

(b) the set $\left\{u_{n}^{2}: n \in \mathbb{N}\right\}$ is relatively compact in $C\left([0, a] ; X_{\alpha}\right)$,

(c) for $j \in\{1, \ldots, N\}$ and $b \in\left(t_{j}, t_{j+1}\right]$, the $\operatorname{set}\left\{u_{\left.n\right|_{\left[b, t_{j+1}\right]} ^{3}}^{3}: n \in \mathbb{N}\right\}$ is relatively compact in $C\left(\left[b, t_{j+1}\right] ; X_{\alpha}\right)$.

Proof. By noting that the number $r$ in the statement of Theorem 2.9 is independent of $n \in \mathbb{N}$, from (2.9) we infer that

$$
\begin{aligned}
\left\|u_{n}\right\|_{\alpha, j} \leq & \left\|u_{n}^{1}\right\|_{\alpha, j}+\left\|u_{n}^{2}\right\|_{\alpha, j}+\left\|u_{n}^{3}\right\|_{\alpha, j} \\
\leq & C_{\alpha}\left\|x_{0}\right\|+C_{\alpha} Q\left\|m_{f}\right\|_{L^{q^{\prime}}([0, a])}\left(\int_{0}^{a} W_{f}\left(\frac{2^{\alpha} r}{s^{\alpha}}\right) d s\right)^{1 / q} \\
& +C_{\alpha} C_{0} \sum_{i=1}^{j}\left(\frac{c_{i}\left(\frac{r}{\delta_{i-1}^{\alpha}}\right) r}{\delta_{i-1}^{\alpha}}+d_{i}\right)
\end{aligned}
$$

which from condition (2.7) implies that $\left\{u_{n}^{i}: i=1,2,3, n \in \mathbb{N}\right\} \subset B_{r}\left(0, \mathcal{P} C_{\alpha}\left(X_{\alpha}\right)\right)$. Moreover, since the estimates (2.10) and (2.11) are independent of $n \in \mathbb{N}$, from (2.11) we have

$$
\left\|u_{n}\right\|_{\mathcal{P} C}(X) \leq\left\|u_{n}^{1}\right\|_{\mathcal{P} C(X)}+\left\|u_{n}^{2}\right\|_{\mathcal{P} C(X)}+\left\|u_{n}^{3}\right\|_{\mathcal{P} C(X)} \leq C_{0}\left\|x_{0}\right\|+C_{0} N \mathfrak{Q}+\frac{C_{0}^{2}}{C_{\alpha}} r,
$$

where $\mathfrak{Q}:=\left\|m_{f}\right\|_{L^{q^{\prime}([0, a])}}\left(\int_{0}^{a} W_{f}^{q}\left(r / \tau^{\alpha}\right) d \tau\right)^{1 / q}$. This completes the proof of (a).

We now prove (b). Let $\Gamma^{2}$ be the map introduced in the proof of Theorem 2.9. From the estimate (2.9) we infer that $\Gamma^{2} B_{r}\left(0, \mathcal{P} C_{\alpha}\left(X_{\alpha}\right)\right) \subset B_{r}\left(0, \mathcal{P} C_{\alpha}\left(X_{\alpha}\right)\right)$, which from 
Steps 1 and 2 permits us to conclude that $\Gamma^{2} B_{r}\left(0, \mathcal{P} C_{\alpha}\left(X_{\alpha}\right)\right)$ is relatively compact in $C\left([0, a] ; X_{\alpha}\right)$. Since the definition of $\Gamma^{2}$ is independent of $n, u_{n}^{2}=\Gamma^{2} u_{n}$ for all $n \in \mathbb{N}$ and $\left\{u_{n}^{2}: n \in \mathbb{N}\right\} \subset B_{r}\left(0, \mathcal{P} C_{\alpha}\left(X_{\alpha}\right)\right)$, we have that $\left\{u_{n}^{2}: n \in \mathbb{N}\right\} \subset \Gamma^{2} B_{r}\left(0, \mathcal{P} C_{\alpha}\left(X_{\alpha}\right)\right)$ which implies that $\left\{u_{n}^{2}: n \in \mathbb{N}\right\}$ is relatively compact in $C\left([0, a] ; X_{\alpha}\right)$. This proves (b).

We divide the proof of (c) into two steps.

Step 1. For $b \in\left(t_{j}, t_{j+1}\right]$, the set $\left\{u_{n}^{3}(s): n \in \mathbb{N}, s \in\left[b, t_{j+1}\right]\right\}$ is relatively compact in $X_{\alpha}$.

Let $t \in\left[b, t_{j+1}\right]$ and $0<\vartheta \leq\left(b-t_{j}\right) / 2$. Since $\left\{u_{n}: n \in \mathbb{N}\right\} \subset B_{r}\left(0, \mathcal{P} C_{\alpha}\left(X_{\alpha}\right)\right)$, from the definition of the norm in $\mathcal{P} C_{\alpha}\left(X_{\alpha}\right)$ we have that $\left\|u_{n}(s-\vartheta)\right\|_{\alpha} \leq r /\left(s-\vartheta-t_{j}\right)^{\alpha} \leq$ $\mu:=2^{\alpha} r /\left(b+t_{j}\right)^{\alpha}$ for all $s \in\left[b, t_{j+1}\right]$. Using this fact, for $n \in \mathbb{N}$ and $t \in\left[b, t_{j+1}\right]$,

$$
\begin{aligned}
(-A)^{\alpha} u_{n}^{3}(t) & =T(\vartheta)(-A)^{\alpha} T\left(\frac{1}{n}\right) \sum_{i=1}^{j} T\left(t-\vartheta-t_{i}\right) I_{i}\left(u\left(t_{i}\right)\right) \\
& =T(\vartheta)(-A)^{\alpha} u_{n}^{3}(t-\vartheta) \in T(\vartheta) B_{\mu}(0, X),
\end{aligned}
$$

which implies that $\left\{(-A)^{\alpha} u_{n}^{3}(s): n \in \mathbb{N}, s \in\left[b, t_{j+1}\right]\right\} \subset T(\vartheta) B_{\mu}(0, X)$. This proves the assertion since $T(\vartheta)$ is compact.

Step 2. For every $b \in\left(t_{j}, t_{j+1}\right]$, the set $\left\{u_{n}^{3}: n \in \mathbb{N}\right\}$ is an equicontinuous subset of $C\left(\left[b, t_{j+1}\right] ; X_{\alpha}\right)$.

Assume that $t \in\left[b, t_{j+1}\right)$. By using the fact that $\left\{(-A)^{\alpha} u_{n}^{3}(t): n \in \mathbb{N}\right\}$ is relatively compact in $X$, for given $\varepsilon>0$ we select $0<\delta<t_{j+1}-t$ such that $\left\|T(s) x-T\left(s^{\prime}\right) x\right\| \leq \varepsilon$ for all $x \in\left\{(-A)^{\alpha} u_{n}^{3}(t): n \in \mathbb{N}\right\}$ and every $s, s^{\prime} \in[0, a]$ with $0<\left|s-s^{\prime}\right|<\delta$. Then, for $0<h<\delta$ and $n \in \mathbb{N}$,

$$
\left\|(-A)^{\alpha} u_{n}^{3}(t+h)-(-A)^{\alpha} u_{n}^{3}(t)\right\|=\left\|(T(h)-I)(-A)^{\alpha} u_{n}^{3}(t)\right\| \leq \varepsilon,
$$

which shows that $\left\{u_{n}^{3}: n \in \mathbb{N}\right\}$ is right equicontinuous at $t \in\left[b, t_{j+1}\right)$.

We now prove the left equicontinuity for the case where $t \in\left(b, t_{j+1}\right]$. Since the set $\left\{(-A)^{\alpha} u_{n}^{3}(s): n \in \mathbb{N}, s \in\left[\left(b+t_{j}\right) / 2, t_{j+1}\right]\right\}$ is relatively compact in $X$ (see Step 1 ), for $\varepsilon>0$ there exists $0<\delta<\left(b-t_{j}\right) / 2$ such that $\|(T(s)-I) x\| \leq \varepsilon$ for all $x \in$ $\left\{(-A)^{\alpha} u_{n}^{3}(s): n \in \mathbb{N}, t \in\left[\left(b+t_{j}\right) / 2, t_{j+1}\right]\right\}$ and each $0<s<\delta$. Then, for $0<h<\delta$ and $p \in \mathbb{N}$ it follows that

$$
\begin{aligned}
& \left\|(-A)^{\alpha} u_{p}^{3}(t-h)-(-A)^{\alpha} u_{p}^{3}(t)\right\|=\left\|(I-T(h))(-A)^{\alpha} u_{n}^{3}(t-h)\right\| \\
& \quad \leq \sup \left\{\left\|(I-T(h))(-A)^{\alpha} u_{n}^{3}(s)\right\|: n \in \mathbb{N}, s \in\left[\frac{b+t_{j}}{2}, t_{j+1}\right]\right\} \leq \varepsilon,
\end{aligned}
$$

which proves that $\left\{u_{n}^{3}: n \in \mathbb{N}\right\}$ is left equicontinuous at $t$.

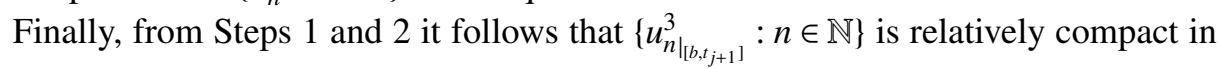
$C\left(\left[b, t_{j+1}\right], X_{\alpha}\right)$. 
In the next lemma, we use the notation introduced in (1.4).

Lemma 2.12. If the assumptions in Theorem 2.9 are fulfilled, then $\left\{u_{n}^{3}: n \in \mathbb{N}\right\}$ is relatively compact in $\mathcal{P C}(X)$.

Proof. To prove this result, we use Lemma 1.1. From Lemma 2.11 we know that $\left\{u_{n}^{i}, u_{n}: i=1,2,3, n \in \mathbb{N}\right\}$ is bounded in $\mathcal{P} C(X)$ and $\left\{u_{\left.n\right|_{\left[b, t_{j+1}\right]} ^{3}}: n \in \mathbb{N}\right\}$ is relatively compact in $C\left(\left[b, t_{j+1}\right] ; X\right)$ for every $j$ and all $b \in\left(t_{j}, t_{j+1}\right]$. Thus, to complete the proof it is sufficient to show that $\left\{\widetilde{\left(u_{n}^{3}\right)_{j}}: n \in \mathbb{N}\right\}$ is right equicontinuous at $t_{j}$ and $\left\{\widetilde{\left(u_{n}^{3}\right)_{j}}\left(t_{j}\right): n \in \mathbb{N}\right\}$ is relatively compact in $X$ for all $j \in\{1, \ldots, N\}$.

Let $j \in\{1, \ldots, N\}$. From Lemma 2.11(b) and (c), the set $P=\left\{u_{n}\left(t_{i}\right): i=1\right.$, $\ldots, N, n \in \mathbb{N}\}$ is relatively compact in $X$, which implies that $Q=\{T(1 / n) x: n \in \mathbb{N}$, $\left.x \in \cup_{i=1}^{N} I_{i}(P)\right\}$ is also relatively compact in $X$. In particular, there exists $R>0$ such that $Q \subset B_{R}(0, X)$.

Step 1. The set $\left.\left\{\widetilde{\left(u_{n}^{3}\right)_{j}}\left(t_{j}\right): n \in \mathbb{N}\right)\right\}$ is relatively compact in $X$.

For $p \in \mathbb{N}$, we have that

$$
\widetilde{\left(u_{p}^{3}\right)_{j}}\left(t_{j}\right)=\sum_{i=1}^{j-1} T\left(t_{j}-t_{i}\right) T\left(\frac{1}{p}\right) I_{i}\left(u_{p}\left(t_{i}\right)\right)+T\left(\frac{1}{p}\right) I_{j}\left(u_{p}\left(t_{j}\right)\right) \in \sum_{i=1}^{j-1} T\left(t_{j}-t_{i}\right) B_{R}(0, X)+Q,
$$

and hence $\left.\left\{\widetilde{\left(u_{n}^{3}\right)_{j}}\left(t_{j}\right): n \in \mathbb{N}\right)\right\} \subset \sum_{i=1}^{j-1} T\left(t_{j}-t_{i}\right) B_{R}(0, X)+Q$. This proves the assertion since the operators $T\left(t_{j}-t_{i}\right)$ are compact.

Step 2. The set $\left.\left\{\widetilde{\left(u_{n}^{3}\right)_{j}}: n \in \mathbb{N}\right)\right\}$ is right equicontinuous at $t=t_{j}$ in the norm of $X$.

Since $\left.\left\{\widetilde{\left(u_{n}^{3}\right)_{j}}\left(t_{j}\right): n \in \mathbb{N}\right)\right\}$ is relatively compact in $X$, for $\varepsilon>0$ there exists $0<\delta<$ $t_{j+1}-t_{j}$ such that $\|(T(s)-I) x\| \leq \varepsilon$ for all $\left.x \in\left\{\widetilde{\left(u_{n}^{3}\right)_{j}}\left(t_{j}\right): n \in \mathbb{N}\right)\right\}$ and each $0<s<\delta$. Then, for $0<h<\delta$ and $p \in \mathbb{N}$,

$$
\begin{aligned}
\left\|\widetilde{\left(u_{p}^{3}\right)_{j}}\left(t_{j}+h\right)-\widetilde{\left(u_{p}^{3}\right)_{j}}\left(t_{j}\right)\right\| & =\left\|T(h) \sum_{i=1}^{j} T\left(t_{j}-t_{i}\right) T\left(\frac{1}{p}\right) I_{i}\left(u_{p}\left(t_{i}\right)\right)-\widetilde{\left(u_{p}^{3}\right)_{j}}\left(t_{j}\right)\right\| \\
& =\left\|(T(h)-I) \widetilde{\left(u_{p}^{3}\right)_{j}}\left(t_{j}\right)\right\| \\
& \leq \sup _{n \in \mathbb{N}}\left\|(T(h)-I)\left(\widetilde{u_{n}^{3}}\right)_{j}\left(t_{j}\right)\right\| \\
& \leq \varepsilon,
\end{aligned}
$$

which shows that $\left.\left\{\widetilde{\left(u_{n}^{3}\right)_{j}}: n \in \mathbb{N}\right)\right\}$ is right equicontinuous at $t=t_{j}$.

From Steps 1 and 2 we infer that $\left\{\widetilde{\left(u_{n}^{3}\right)_{j}}: n \in \mathbb{N}\right\}$ is relatively compact in $C\left(\left[t_{j}, t_{j+1}\right], X\right)$. This completes the proof.

We are now in a position to prove the main result of this work.

Theorem 2.13. Suppose that the assumptions in Theorem 2.9 are fulfilled. Then there exists a mild solution $u \in \mathcal{P C}_{\alpha}\left(X_{\alpha}\right)$ of the impulsive problem (1.1)-(1.3). 
Proof. From Lemmas 2.11 and 2.12 there exist a subsequence $\left(u_{n_{i}}\right)_{i \in \mathbb{N}}$ of $\left(u_{n}\right)_{n \in \mathbb{N}}$ and $u \in \mathcal{P C}(X)$ such that $\left\|u_{n_{i}}-u\right\|_{\mathcal{P} C(X)} \rightarrow 0$ as $i \rightarrow \infty$ and $u_{\left.n_{i}\right|_{\left[b, t_{j}\right]}} \rightarrow u$ in $C\left(\left[b, t_{j+1}\right], X_{\alpha}\right)$ for all $j \in\{0, \ldots, N\}$ and every $b \in\left(t_{j}, t_{j+1}\right]$. Moreover, from Lemma 2.11 we know that $\left(t-t_{j}\right)^{\alpha}\left\|(-A)^{\alpha} u_{n}(t)\right\| \leq r$ for all $n \in \mathbb{N}, j \in\{0, \ldots, N\}$ and each $t \in\left(t_{j}, t_{j+1}\right]$, which implies that $\left(t-t_{j}\right)^{\alpha}\left\|(-A)^{\alpha} u(t)\right\| \leq r$ for all $t \in\left(t_{j}, t_{j+1}\right]$ and every $j \in\{0, \ldots, N\}$. This proves that $u \in B_{r}\left(0, \mathcal{P} C_{\alpha}\left(X_{\alpha}\right)\right)$. Finally, a standard application of the Lebesgue dominated convergence theorem allows us to conclude that $u(\cdot)$ is a mild solution.

Next, we consider two easy consequences of Theorem 2.13.

Corollary 2.14. Assume that $\mathrm{H}_{2}$ and $\mathrm{H}_{3}$ are satisfied, the semigroup $(T(t))_{t \geq 0}$ is compact and there are positive constants $\gamma \in(0,1 / \alpha q), \mu_{i}, \tilde{c}_{i} i=1, \ldots, N$, such that $W_{f}(s)=s^{\gamma}$ and $c_{i}(s)=\tilde{c}_{i} s^{\mu_{i}}$ for all $s \geq 0$ and every $i=1, \ldots, N$. Suppose that $\lim _{h \downarrow 0}\left\|m_{f}\right\|_{L^{q^{\prime}([d, d+h])}} h^{1 / q-\alpha(\gamma+1)}=0$ for all $d \in[0, a)$ and $\lim _{h \downarrow 0}\left\|m_{f}\right\|_{L^{q^{\prime}([c-h, c])}} h^{1 / q-\alpha(\gamma+1)}$ $=0$ uniformly for $c$ in compact subsets of $(0, a]$ and there is $r>0$ such that

$$
C_{\alpha}\left(\left\|x_{0}\right\|+Q\left\|m_{f}\right\|_{L^{q^{\prime}([0, a])}} \frac{2^{\alpha \gamma} a^{1 / q-\alpha \gamma}}{(1-q \alpha \gamma)^{1 / q}} r^{\gamma}+C_{0} \sum_{i=1}^{N}\left(\frac{\tilde{c}_{i} r^{\mu_{i}+1}}{\delta_{i-1}^{\alpha\left(\mu_{i}+1\right)}}+d_{i}\right)\right) \leq r .
$$

Then there exists a mild solution $u \in \mathcal{P} C_{\alpha}\left(X_{\alpha}\right)$ of (1.1)-(1.3). In particular, if $1 / q-\alpha(\gamma+1)>0$ and $r$ is as above, then there exits a mild solution $u \in \mathcal{P} C_{\alpha}\left(X_{\alpha}\right)$.

Corollary 2.15. Assume that conditions $\mathrm{H}_{2}$ and $\mathrm{H}_{3}$ are satisfied, there are constants $\tilde{c}_{i}, i=1, \ldots, N$, such that $c_{i}(s) \leq \tilde{c}_{i}$ for all $i=1, \ldots, N$ and each $s>0$, the semigroup $(T(t))_{t \geq 0}$ is compact and the function $\tau \rightarrow W_{f}^{q}\left(1 / \tau^{\alpha}\right)$ is integrable on $[0, a]$. Suppose, in addition, that $\lim _{h \downarrow 0}\left\|m_{f}\right\|_{L^{q^{\prime}}([c, c+h])}\left(1 / h^{\alpha}\right)\left(\int_{0}^{h} W_{f}^{q}\left(1 / s^{\alpha}\right) d s\right)^{1 / q}=0$ for all $c \in[0, a)$, $\lim _{h \rightarrow 0}\left\|m_{f}\right\|_{L^{q^{\prime}([d-h, d])}}\left(1 / h^{\alpha}\right)\left(\int_{0}^{h} W_{f}^{q}\left(\left(1 / s^{\alpha}\right)\right) d s\right)^{1 / q}=0$ for each $d \in(0, a]$ and

$$
C_{\alpha} Q\left\|m_{f}\right\|_{L^{q^{\prime}}([0, a])} \limsup _{r \rightarrow \infty} \frac{1}{r}\left(\int_{0}^{a} W_{f}^{q}\left(\frac{2^{\alpha} r}{s^{\alpha}}\right) d s\right)^{1 / q}+C_{0} \sum_{i=1}^{N} \frac{\tilde{c}_{i}}{\delta_{i-1}^{\alpha}}<1 .
$$

Then there exists a mild solution $u \in \mathcal{P} C_{\alpha}\left(X_{\alpha}\right)$ of (1.1)-(1.3). In particular, if $W_{f}(\cdot)$ is bounded and $C_{\alpha} C_{0} \sum_{i=1}^{N} \tilde{c}_{i} / \delta_{i-1}^{\alpha}<1$, then there exits a mild solution $u \in \mathcal{P} C_{\alpha}\left(X_{\alpha}\right)$ of (1.1)-(1.3).

We complete this section by studying the existence of a classical solution. We assume that the conditions in Theorem 2.6 are satisfied and that $u \in \mathcal{P} C_{\alpha}\left(X_{\alpha}\right)$ is a mild solution of (1.1)-(1.3). For completeness, we note that a function $v \in C([c, d] ; X)$ is called a classical solution of

$$
\begin{aligned}
w^{\prime}(t) & =A w(t)+\xi(t), \quad t \in(c, d],\left(\xi \in L^{1}([c, d] ; X)\right) \\
w(c) & =z \in X
\end{aligned}
$$

if $v(t) \in D(A)$ for all $t \in(c, d], A v(\cdot)$ is continuous on $(c, d], v(c)=z$ and $v(\cdot)$ satisfies the equation (2.12). 
In the following theorem we establish conditions under which the mild solution $u(\cdot)$ of (1.1)-(1.3) is a classical solution.

Proposition 2.16. Assume that there are $\beta \in(0,1)$ and a function $\mathcal{K}_{f} \in C\left([0, \infty) ; \mathbb{R}^{+}\right)$ such that

$$
\|f(t, x)-f(s, y)\| \leq \mathcal{K}_{f}(l)\left(|t-s|^{\beta}+\left\|(-A)^{\alpha} x-(-A)^{\alpha} y\right\|\right),
$$

for all $t, s \in[0, a], x, y \in B_{l}\left(0, X_{\alpha}\right)$ and each $l>0$. Then $u(\cdot)$ is a classical solution.

Proof. To prove the assertion, we show that each function $\widetilde{u}_{j}$ (see (1.4)) is a classical solution of

$$
z^{\prime}(t)=A z(t)+f(t, z(t)), \quad t \in\left(t_{j}, t_{j+1}\right], \quad z\left(t_{j}\right)=u\left(t_{j}^{+}\right)
$$

To begin, we study the case $j=0$. Let $a_{1} \in\left(0, t_{1}\right)$. We affirm that $u\left(a_{1}\right) \in X_{\mu}$ for all $\mu \in(0,1)$. To prove our claim, we note that $u_{\left[a_{1} / 2, t_{1}\right]} \in C\left(\left[a_{1} / 2, t_{1}\right], X_{\alpha}\right)$, which implies that the function $\|f(\cdot, u(\cdot))\|$ is bounded on $\left[a_{1} / 2, t_{1}\right]$. Using this fact, for $\mu \in(0,1)$ we see that

$$
\begin{aligned}
\left\|(-A)^{\mu} u\left(a_{1}\right)\right\| & \leq\left\|(-A)^{\mu} T\left(a_{1}-\frac{a_{1}}{2}\right) u\left(\frac{a_{1}}{2}\right)\right\|+\int_{a_{1} / 2}^{a_{1}}\left\|(-A)^{\mu} T\left(a_{1}-\tau\right) f(\tau, u(\tau))\right\| d \tau \\
& \leq \frac{2^{\mu} C_{\mu}}{a_{1}^{\mu}}\left\|u\left(\frac{a_{1}}{2}\right)\right\|+C_{\mu}\|f(\cdot, u(\cdot))\|_{C\left(\left[a_{1} / 2, t_{1}\right] ; X\right)} \frac{\left(\frac{a_{1}}{2}\right)^{1-\mu}}{1-\mu},
\end{aligned}
$$

which shows $\left\|(-A)^{\mu} u\left(a_{1}\right)\right\|$ is finite. This proves that $u\left(a_{1}\right) \in X_{\mu}$.

Let $r_{1}>0$ and $k \in \mathbb{N}$ such that $\|u\|_{C\left(\left[a_{1} / 2, t_{1}\right] ; X_{\alpha}\right)} \leq r_{1}$ and $\vartheta=C_{\alpha} \mathcal{K}_{f}\left(r_{1}\right) \times$ $b^{1-\alpha} /(1-\alpha)<1$ where $b=\left(t_{1}-a_{1}\right) / k$. Under these conditions, for $\mu_{1} \in(\alpha, 1), t \in$ $\left[a_{1}, a_{1}+b\right)$ and $h>0$ such that $t+h \in\left[a_{1}, a_{1}+b\right]$,

$$
\begin{aligned}
& \left\|(-A)^{\alpha} u(t+h)-(-A)^{\alpha} u(t)\right\| \\
& \leq\left\|(-A)^{\alpha} T\left(t-a_{1}\right)\left(T(h) u\left(a_{1}\right)-u\left(a_{1}\right)\right)\right\| \\
& \quad+\int_{a_{1}}^{a_{1}+h}\left\|(-A)^{\alpha} T(t+h-\tau) f(\tau, u(\tau))\right\| d \tau \\
& \quad+\int_{a_{1}}^{t}\left\|(-A)^{\alpha} T(t-\tau)\right\|\|f(\tau+h, u(\tau+h))-f(\tau, u(\tau))\| d \tau \\
& \leq\left\|T\left(t-a_{1}\right)\right\| \int_{0}^{h}\left\|(-A)^{1+\alpha-\mu_{1}} T(\tau)(-A)^{\mu_{1}} u\left(a_{1}\right)\right\| d \tau \\
& \quad+C_{\alpha}\|f(\cdot, u(\cdot))\|_{C\left(\left[a_{1}, t_{1}\right] ; X\right)} \int_{a_{1}}^{a_{1}+h} \frac{d \tau}{\left(a_{1}+h-\tau\right)^{\alpha}} \\
& \quad+\int_{a_{1}}^{t} \frac{C_{\alpha} \mathcal{K}_{f}\left(r_{1}\right)}{(t-\tau)^{\alpha}}\left(h^{\beta}+\left\|(-A)^{\alpha} u(\tau+h)-(-A)^{\alpha} u(\tau)\right\|\right) d \tau
\end{aligned}
$$




$$
\begin{aligned}
\leq & C_{0} C_{1+\alpha-\mu_{1}}\left\|(-A)^{\mu_{1}} u\left(a_{1}\right)\right\| \int_{0}^{h} \frac{d \tau}{\tau^{1+\alpha-\mu_{1}}} \\
& +C_{\alpha}\|f(\cdot, u(\cdot))\|_{C\left(\left[a_{1}, t_{1}\right] ; X\right)} \frac{h^{1-\alpha}}{1-\alpha} \\
& +C_{\alpha} \mathcal{K}_{f}\left(r_{1}\right) h^{\beta} \frac{a^{1-\alpha}}{1-\alpha}+C_{\alpha} \mathcal{K}_{f}\left(r_{1}\right) \int_{a_{1}}^{t} \frac{\left\|(-A)^{\alpha} u(\tau+h)-(-A)^{\alpha} u(\tau)\right\|}{(t-\tau)^{\alpha}} d \tau \\
\leq & D_{1} h^{\mu_{2}}+C_{\alpha} \mathcal{K}_{f}\left(r_{1}\right) \frac{b^{1-\alpha}}{1-\alpha} \sup _{\theta \in\left[a_{1}, b\right]}\left\|(-A)^{\alpha} u(\tau+h)-(-A)^{\alpha} u(\tau)\right\|,
\end{aligned}
$$

where $\mu_{2}=\min \left\{\mu_{1}-\alpha, 1-\alpha, \beta\right\}$ and $D_{1}$ is a positive constant independent of $t \in\left[a_{1}, a_{1}+b\right]$. From this inequality we infer that $\left\|(-A)^{\alpha} u(t+h)-(-A)^{\alpha} u(t)\right\| \leq$ $D_{1} h^{\mu_{2}} /(1-\vartheta)$ for all $t \in\left(a_{1}, a_{1}+b\right], \quad u \in C^{\mu_{2}}\left(\left[a_{1}, a_{1}+b\right] ; X_{\alpha}\right)$ and $f(\cdot, u(\cdot)) \in$ $C^{\mu_{2}}\left(\left[a_{1}, a_{1}+b\right] ; X\right)$. Now, from [20, Theorem 4.3.2] it follows that the mild solution of the problem

$$
z^{\prime}(t)=A z(t)+f(t, u(t)), \quad t \in\left[a_{1}, a_{1}+b\right], \quad z\left(a_{1}\right)=u\left(a_{1}\right),
$$

is a classical solution and from the uniqueness of mild solutions of (2.14) we obtain that $u=w$ on $\left[a_{1}, a_{1}+b\right], A u_{\left.\right|_{\left(a_{1}, a_{1}+b\right]}}$ is continuous on $\left(a_{1}, b\right], u\left(t_{1}+b\right) \in D(A)$ and $u_{\left[a_{1}, a_{1}+b\right]}$ is a classical solution of

$$
z^{\prime}(t)=A z(t)+f(t, z(t)), \quad t \in\left[a_{1}, a_{1}+b\right], z\left(a_{1}\right)=u\left(a_{1}\right) .
$$

Arguing as above, we can show that $u_{\left[a_{1}+b, a_{1}+2 b\right]}$ is a classical solution of

$$
z^{\prime}(t)=A z(t)+f(t, z(t)), \quad t \in\left(a_{1}+b, a_{1}+2 b\right], z\left(a_{1}+b\right)=u\left(a_{1}+b\right),
$$

$A u_{\left[a_{1}+b, a_{1}+2 b\right]} \in C\left(\left[a_{1}+b, a_{1}+2 b\right] ; X\right)$ and $u\left(a_{1}+2 b\right) \in D(A)$. Continuing this procedure, we can prove that $u(\cdot)$ is a classical solution of

$$
z^{\prime}(t)=A z(t)+f(t, z(t)), \quad t \in\left(a_{1}, t_{1}\right], z\left(a_{1}\right)=u\left(a_{1}\right) .
$$

Since $a_{1}$ is arbitrary, from the above steps it follows that $u_{\left[0, t_{1}\right]}$ is a classical solution of

$$
z^{\prime}(t)=A z(t)+f(t, z(t)), \quad t \in\left(0, t_{1}\right], z(0)=x_{0} .
$$

Repeating the process described on each interval $\left[t_{j}, t_{j+1}\right]$, we prove that $\widetilde{u}_{j}(\cdot)$ is a classical solution of (1.1)-(1.3). This completes the proof.

\section{Applications}

In this section, $X=L^{2}([0, \pi])$ and $A: D(A) \subset X \rightarrow X$ is the operator given by $A x=x^{\prime \prime}$ with domain $D(A):=\left\{x \in X: x^{\prime \prime} \in X, \quad x(0)=x(\pi)=0\right\}$. It is well known that $A$ is the generator of an analytic, compact semigroup $(T(t))_{t \geq 0}$ on $X$, and $A$ has discrete spectrum with eigenvalues $-n^{2}, n \in \mathbb{N}$, and associated normalised 
eigenvectors $z_{n}(\xi)=(2 / \pi)^{1 / 2} \sin (n \xi)$. Moreover, the set of functions $\left\{z_{n}: n \in \mathbb{N}\right\}$ is an orthonormal basis of $X, T(t) x=\sum_{n=1}^{\infty} e^{-n^{2} t}\left\langle x, z_{n}\right\rangle z_{n}$ and $\|T(t)\| \leq e^{-t}$ for all $x \in X$ and every $t \geq 0$. In addition, $(-A)^{-1 / 2} x=\sum_{n=1}^{\infty}(1 / n)\left\langle x, z_{n}\right\rangle z_{n}$ for $x \in X,(-A)^{1 / 2} x=$ $\sum_{n=1}^{\infty} n\left\langle x, z_{n}\right\rangle z_{n}$ for $x \in D\left((-A)^{1 / 2}\right)=\left\{x \in X: \sum_{n=1}^{\infty} n\left\langle x, z_{n}\right\rangle z_{n} \in X\right\},\left\|(-A)^{-1 / 2}\right\|_{\mathcal{L}(X)}=1$ and $\left\|(-A)^{1 / 2} T(t)\right\| \leq \frac{1}{\sqrt{2}} e^{-t / 2} t^{-1 / 2}$ for all $t>0$.

To begin, we study the existence of solutions for the impulsive problem

$$
\begin{aligned}
\frac{\partial}{\partial t} w(t, \xi) & =\frac{\partial^{2}}{\partial \xi^{2}} w(t, \xi)+\mu(t) w(t, \xi), \quad(t, \xi) \in[0, a] \times[0, \pi] \\
w(t, 0) & =w(t, \pi)=0, \quad t \in[0, a] \\
w(0, \xi) & =z(\xi), \quad \xi \in[0, \pi] \\
\Delta w\left(t_{i}, \xi\right) & =\theta_{i} \frac{\partial}{\partial \xi} w\left(t_{i}, \xi\right), \quad \xi \in[0, \pi], i=1, \ldots, N
\end{aligned}
$$

where $0<t_{1}<\cdots<t_{N} \leq a, \theta_{i}$ are fixed real numbers, $z \in X$ and $\mu:[0, a] \rightarrow \mathbb{R}$ is a suitable function.

To represent (3.1)-(3.4) in the abstract form (1.1)-(1.3), we assume that $\mu \in$ $L^{q^{\prime}}([0, a] ; \mathbb{R})$ for some $q \in[1,2)$, where $q^{\prime}$ denotes the conjugate of $q\left(1 / q+1 / q^{\prime}=1\right)$. We introduce the functions $f:[0, a] \times X \rightarrow X$ and $I_{i}: X_{1 / 2} \rightarrow X$ given by $f(t, x)(\xi)=$ $\mu(t) x(\xi)$ and $I_{i}(x)=\theta_{i}(\partial x / \partial \xi)$. It is easy to see that $I_{i} \in \mathcal{L}\left(X_{1 / 2}, X\right)$ for all $i$ and there exists $C>0$ such that $\left\|I_{i}\right\|_{\mathcal{L}\left(X_{1 / 2}, X\right)} \leq C\left|\theta_{i}\right|$ for all $i=1, \ldots, N$. We also note $\|f(t, x)\| \leq m_{f}(t) W_{f}\left(\left\|(-A)^{\alpha} x\right\|\right)$ with $m_{f}(t)=|\mu(t)|$ and $W_{f}(s)=|s|$.

In the next result, we say that $u \in \mathcal{P C}(X)$ is a mild solution (respectively, a classical solution) of (3.1)-(3.4) if $u(\cdot)$ is a mild solution (respectively, a classical solution) of the associated abstract problem (1.1)-(1.3). In this result, and in the remainder of this section, $Q:=N+2^{1 / 2+1 / q} /|1-q / 2|^{1 / q}$.

Proposition 3.1. Assume that

$$
\frac{1}{\sqrt{2}}\left(Q \frac{\sqrt{2} a^{1 / q-1 / 2}}{|1-q / 2|^{1 / q}}\|\mu\|_{L^{q^{\prime}}([0, a])}+C \sum_{i=1}^{N} \frac{\left|\theta_{i}\right|}{\delta_{i-1}^{1 / 2}}\right)<1 .
$$

Then there exists a unique mild solution $u \in \mathcal{P} C_{1 / 2}\left(X_{1 / 2}\right)$ of (3.1)-(3.4). If, in addition, $\mu \in C^{\beta}([0, a] ; \mathbb{R})$ for some $\beta \in(0,1)$, then $u(\cdot)$ is a classical solution.

Proof. The existence of a mild solution $u \in \mathcal{P} C_{1 / 2}\left(X_{1 / 2}\right)$ follows from Corollary 2.7. If $\mu \in C^{\beta}([0, a] ; \mathbb{R})$, then the function $f(\cdot)$ satisfies the assumptions of Proposition 2.16 which implies that $u(\cdot)$ is a classical solution.

Similar to the previous example, in the next problems we say that $u \in \mathcal{P C}(X)$ is a mild solution if $u(\cdot)$ is a mild solution of the associated problem (1.1)-(1.3). Consider 
the problem

$$
\begin{aligned}
\frac{\partial}{\partial t} w(t, \xi) & =\frac{\partial^{2}}{\partial \xi^{2}} w(t, \xi)+\mu(t)|w(t, \xi)|^{\gamma}, \quad(t, \xi) \in[0, a] \times[0, \pi], \\
w(t, 0) & =w(t, \pi)=0, \quad t \in[0, a], \\
w(0, \xi) & =z(\xi), \quad \xi \in[0, \pi], \\
\triangle w\left(t_{i}, \xi\right) & =\theta_{i} \frac{\partial}{\partial \xi} w\left(t_{i}, \xi\right), \quad \xi \in[0, \pi],
\end{aligned}
$$

where $t_{i}, \theta_{i}, \mu(\cdot)$ are as above and $\gamma \in(1,2)$. In Proposition 3.2 we establish the existence of a mild solution for (3.5)-(3.8) via Corollary 2.15.

Proposition 3.2. Assume that $C \sum_{i=1}^{N} \theta_{i} \delta_{i-1}^{-1 / 2}<1, \lim _{h \downarrow 0}\|\mu\|_{L^{q^{\prime}([d, d+h])}} h^{1 / q-\alpha(\gamma+1)}=0$ for all $d \in[0, a)$ and $\lim _{h \downarrow 0}\|\mu\|_{L^{q^{\prime}([c-h, c])}} h^{1 / q-\alpha(\gamma+1)}=0$ uniformly for $c$ in compact subsets of $(0, a]$. Then there exists a mild solution $u \in \mathcal{P} C_{1 / 2}\left(X_{1 / 2}\right)$ of (3.5)-(3.8). In particular, if $q \in(1,2 /(\gamma+1))$ and $C \sum_{i=1}^{N} \theta_{i} \delta_{i-1}^{-1 / 2}<1$, then there exists a mild solution for (3.5)-(3.8).

We finish this section by studying the existence of solutions for the problem

$$
\begin{aligned}
\frac{\partial}{\partial t} w(t, \xi) & =\frac{\partial^{2}}{\partial \xi^{2}} w(t, \xi)+\mu(t) w(t, \xi), \quad(t, \xi) \in[0, a] \times[0, \pi], \\
w(t, 0) & =w(t, \pi)=0, \quad t \in[0, a], \\
w(0, \xi) & =z(\xi), \quad \xi \in[0, \pi], \\
\Delta w\left(t_{i}, \xi\right) & =\theta_{i} w\left(t_{i}, \xi\right)\left|w\left(t_{i}, \xi\right)\right|^{\gamma_{i}}, \quad \xi \in[0, \pi],
\end{aligned}
$$

where $\mu, \theta_{i}, \mu$ are as above and $\gamma_{i} \in(0,1)$ for all $i=1, \ldots, N$.

To treat this system, we define the functions $I_{i}: X_{1 / 2} \rightarrow X$ by $I_{i}(x)=\theta_{i} x|x|^{\gamma_{i}}$. The functions $I_{i}$ are well defined and there exists $C>0$ such that $\left\|I_{i} x\right\| \leq C l^{\gamma_{i}}\left\|(-A)^{1 / 2} x\right\|$ for all $x \in B_{l}\left(0, X_{1 / 2}\right)$ and every $l>0$. The next result follows from Theorem 2.6.

Proposition 3.3. Assume that the above conditions are satisfied and there are $r>0$ and $\Theta \in(0,1)$ such that

$$
\frac{1}{\sqrt{2}}\left(Q \frac{\sqrt{2} a^{1 / q-1 / 2}}{|1-q / 2|^{1 / q}}\|\mu\|_{L^{q^{\prime}([0, a])}}+C \sum_{i=1}^{N} \frac{\theta_{i} r^{\gamma_{i}}}{\delta_{i-1}^{\left(\gamma_{i}+1\right) / 2}}\right)<\Theta .
$$

If $\|z\|<\sqrt{2} r(1-\Theta)$, then there exists a unique mild solution of system (3.9)-(3.12).

\section{References}

[1] N. Abada, M. Benchohra and H. Hammouche, 'Existence and controllability results for nondensely defined impulsive semilinear functional differential inclusions', J. Differential Equations 246(10) (2009), 3834-3863.

[2] A. Anguraj and K. Karthikeyan, 'Existence of solutions for impulsive neutral functional differential equations with nonlocal conditions', Nonlinear Anal. 70(7) (2009), 2717-2721. 
[3] L. Barreira and C. Valls, 'Lyapunov regularity of impulsive differential equations', J. Differential Equations 249 (2010), 1596-1619.

[4] M. Benchohra, J. Henderson and S. Ntouyas, Impulsive Differential Equations and Inclusions, Contemporary Mathematics and its Applications, 2 (Hindawi, New York, 2006).

[5] Y.-K. Chang, A. Anguraj and K. Karthikeyan, 'Existence for impulsive neutral integrodifferential inclusions with nonlocal initial conditions via fractional operators', Nonlinear Anal. 71(10) (2009), 4377-4386.

[6] Y.-K. Chang, V. Kavitha and M. M. Arjunan, 'Existence results for impulsive neutral differential and integrodifferential equations with nonlocal conditions via fractional operators', Nonlinear Anal. Hybrid Syst. 4(1) (2010), 32-43.

[7] J. Chu and J. J. Nieto, 'Impulsive periodic solutions of first-order singular differential equations', Bull. Lond. Math. Soc. 40(1) (2008), 143-150.

[8] Z. Fan and G. Li, 'Existence results for semilinear differential equations with nonlocal and impulsive conditions', J. Funct. Anal. 258(5) (2010), 1709-1727.

[9] X. Fu and Y. Cao, 'Existence for neutral impulsive differential inclusions with nonlocal conditions', Nonlinear Anal. 68(12) (2008), 3707-3718.

[10] D. Franco, E. Liz, J. J. Nieto and Y. V. Rogovchenko, 'A contribution to the study of functional differential equations with impulses', Math. Nachr. 218 (2000), 49-60.

[11] M. Frigon and D. O'Regan, 'Existence results for first-order impulsive differential equations', J. Math. Anal. Appl. 193(1) (1995), 96-113.

[12] M. Frigon and D. O'Regan, 'First order impulsive initial and periodic problems with variable moments', J. Math. Anal. Appl. 233(2) (1999), 730-739.

[13] E. Hernández and D. O'Regan, 'On a new class of abstract impulsive differential equations', Proc. Amer. Math. Soc. 141(5) (2013), 1641-1649.

[14] C. Kou, S. Zhang and S. Wu, 'Stability analysis in terms of two measures for impulsive differential equations', J. London Math. Soc. (2) 66(1) (2002), 142-152.

[15] A. Lin and L. Hu, 'Existence results for impulsive neutral stochastic functional integro-differential inclusions with nonlocal initial conditions', Comput. Math. Appl. 59(1) (2010), 64-73.

[16] J. H. Liu, 'Nonlinear impulsive evolution equations', Dynam. Contin. Discrete Impuls. Systems 6(1) (1999), 77-85.

[17] J. J. Nieto and D. O'Regan, 'Variational approach to impulsive differential equations', Nonlinear Anal. Real World Appl. 10(2) (2009), 680-690.

[18] Y. V. Rogovchenko, 'Impulsive evolution systems: main results and new trends', Dynam. Contin. Discrete Impuls. Systems 3(1) (1997), 57-88.

[19] Y. V. Rogovchenko, 'Nonlinear impulse evolution systems and applications to population models', J. Math. Anal. Appl. 207(2) (1997), 300-315.

[20] A. Pazy, Semigroups of Linear Operators and Applications to Partial Differential Equations, Applied Mathematical Sciences, 44 (Springer, New York, 1983).

[21] Z. Yan, 'Existence of solutions for nonlocal impulsive partial functional integrodifferential equations via fractional operators', J. Comput. Appl. Math. 235(8) (2011), 2252-2262.

[22] J. S. Yu and X. H. Tang, 'Global attractivity in a delay population model under impulsive perturbations', Bull. London Math. Soc. 34(3) (2002), 319-328.

EDUARDO HERNÁNDEZ, Departamento de Computação e Matemática, Faculdade de Filosofia Ciêcias e Letras de Ribeirão Preto, Universidade de São Paulo, CEP14040-901 Ribeirão Preto, SP, Brazil e-mail: lalohm@ffclrp.usp.br

DONAL O'REGAN, Department of Mathematics, National University of Ireland, Galway, Ireland e-mail: donal.oregan@nuigalway.ie 